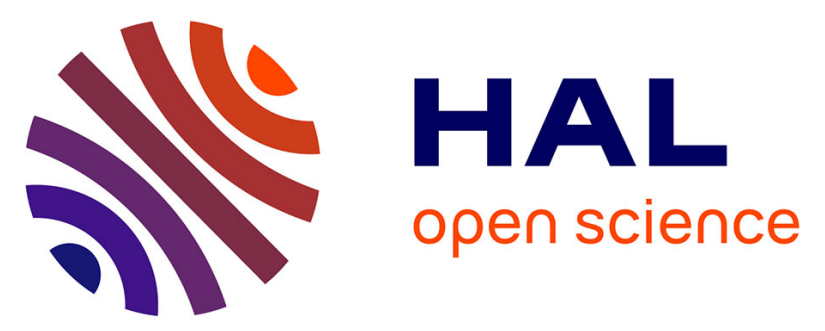

\title{
Géoarchéologie du site antique de Molesme en vallée de Laigne (Côte-d'Or) : mise en évidence de l'impact anthropique sur la sédimentation alluviale
}

Christophe Petit, Christian Camerlynck, Eline Deweirdt, Christophe Durlet, Jean-Pierre Garcia, Émilie Gauthier, Vincent Ollive, Hervé Richard, Patrice Wahlen

\section{To cite this version:}

Christophe Petit, Christian Camerlynck, Eline Deweirdt, Christophe Durlet, Jean-Pierre Garcia, et al.. Géoarchéologie du site antique de Molesme en vallée de Laigne (Côte-d'Or) : mise en évidence de l'impact anthropique sur la sédimentation alluviale. Gallia - Archéologie de la France antique, 2006, 63, pp.263-281. 10.3406/galia.2006.3298 . hal-01911071

\section{HAL Id: hal-01911071 \\ https://hal.science/hal-01911071}

Submitted on 8 Jan 2020

HAL is a multi-disciplinary open access archive for the deposit and dissemination of scientific research documents, whether they are published or not. The documents may come from teaching and research institutions in France or abroad, or from public or private research centers.
L'archive ouverte pluridisciplinaire HAL, est destinée au dépôt et à la diffusion de documents scientifiques de niveau recherche, publiés ou non, émanant des établissements d'enseignement et de recherche français ou étrangers, des laboratoires publics ou privés.

\section{(ㅇ)(1) $\$$}

Distributed under a Creative Commons Attribution - NonCommercial - NoDerivatives $\mid 4.0$ 


\title{
GÉOARCHÉOLOGIE DU SITE ANTIQUE DE MOLESME EN VALLÉE DE LAIGNE (CÔTE-D'OR)
}

\author{
Mise en évidence de limpact anthropique \\ sur la sédimentation alluviale
}

\author{
Christophe PETIT ${ }^{1}$, Christian CAMERLYNCK ${ }^{2}$, Eline DEWEIRDT ${ }^{1}$, \\ Christophe DURLET ${ }^{3}$, Jean-Pierre GARCIA ${ }^{3}$, Émilie GAUTHIER ${ }^{4}$, \\ Vincent OLLIVE ${ }^{3}$, Hervé RICHARD ${ }^{4}$, Patrice WAHLEN ${ }^{5}$
}

\begin{abstract}
Mots-clés. Bourgogne, bassin versant, crues, érosion des sols, impact anthropique, géoarchéologie, paléoenvironnement.
Résumé. Les conclusions issues de l'expérience menée dans la vallée de la Laigne mettent en évidence la pertinence de la démarche géoarchéologique menée à deux échelles d'investigation. La première, conduite sur l'ensemble de la plaine alluviale, permet de recueillir les données générales qui caractérisent le fonctionnement spécifique de ce bassin versant pendant la période holocène: détermination des séquences sédimentaires, végétales et des phases d'occupation. La seconde est celle d'une démarche géoarchéologique menée à l'échelle d'un site-test: elle met en évidence et date le plus précisément possible des interactions entre événements naturels et activités humaines, interactions révélatrices des mutations de l'ensemble du bassin versant. Le contrôle anthropique de la sédimentation alluviale au cours $d u I^{e r}$ s., c'est-à-dire lors de la phase de romanisation de ce terroir, est ainsi clairement mis en évidence.
\end{abstract}

Key-words. Burgundy, catchment basin, flood, soil erosion, anthropogenic impact, geoarchaeology, palaeoenvironment.

Abstract. The conclusions from the research project in the Laigne valley show the relevance of the geoarchaeological approach conducted on two scales of investigation. The first, over the entire alluvial plain, allows general data to be collected which characterise the specific functions of each catchment basin during the Holocene period: determination of sedimentary sequences, evolution of vegetation and phases of human occupation. The second is a geoarchaeological approach on the scale of a test site: it demonstrates and dates as precisely as possible interactions between natural events and human activities which reveal changes to the entire catchment basin.

Translation: Isabelle FAUDUET

Schlagwörter. Bourgogne, Flußsystem, Hochwasser, Bodenerosion, anthropogene Auswirkungen, Geoarchäologie, Paläoumwelt.

Zusammenfassung. Die Rückschlüsse aus unserer Fallstudie zum Tal der Laigne stellen die Stichhaltigkeit der geoarchäologischen Methode heraus, die auf zwei Ebenen der Untersuchung Anwendung fand. In einem ersten Schritt werden, auf die Gesamtheit der Schwemmebene bezogen, die allgemeinen Daten ermittelt, anhand derer sich die spezifische Funktionsweise dieses Flußsystems während des Holozäns charakterisieren läßt (Bestimmung von Sedimentabfolgen, Vegetationsentwicklung und Besiedlungsphasen). In einem

1. UMR 5594 du CNRS: «Archéologie, cultures et sociétés », Université de Bourgogne, 6 boulevard Gabriel, F-21000 Dijon. Courriels: christophe. petit@u-bourgogne.fr; eline.deweirdt@wanadoo.fr

2. UMR 7619 du CNRS: «Laboratoire de géophysique appliquée », tour 25, Université de Paris-VI, F-75008 Paris. Courriel: camerl@ccr.jussieu.fr 3. UMR 5561 du CNRS: «Biogéosciences», Université de Bourgogne, 6 boulevard Gabriel, F-21000 Dijon. Courriels: christophe.durlet @u-bourgogne.fr; jpgarcia@u-bourgogne.fr; vincent.ollive@u-bourgogne.fr

4. UMR 6565 du CNRS: «Laboratoire de chronoécologie », UFR sciences et techniques, Université de Franche-Comté, 16 route de Gray, F-25030 Besançon Cedex. Courriels: herve.richard@univ-fcomte.fr; emilie.gauthier@univ-fcomte.fr

5. Rue de l'Église, F-89800 Saint-Cyr-les-Colons. Courriel: patrice.wahlen@orange.fr 
zweiten Schritt steht die lokale Gegenprobe im Vordergrund. Dabei geht es um die Identifizierung und möglichst exakte Datierung der Interaktionen zwischen Naturgeschehen und menschlichen Aktivitäten, die letztlich über die Veränderungsprozesse im gesamten Flußsystem Aufschluß geben. Damit wird der allesentscheidende Einfluß des Menschen auf die alluviale Sedimentation im Verlauf des 1. Jahrh. n. Chr. deutlich herausgestellt.

Übersetzung: Stefan WIRTH

La démarche entreprise depuis 1996 dans la vallée de la Laigne a pour objectif la mise en évidence des interactions entre milieux naturels et activités anthropiques; c'est dans cette perspective qu'une approche combinant géologie et archéologie a été mise en œuvre dans l'étude de ce bassin versant de Bourgogne du Nord. Une étroite collaboration s'est alors instaurée entre historiens, archéologues et géologues, dans le cadre d'une recherche visant à la reconstitution des paysages historiques et de leur évolution, de la Protohistoire à nos jours et ce à différentes échelles spatiales. C'est pourquoi, situé immédiatement en bordure de la rivière, le site laténien puis gallo-romain dit de Sur-lesCreux à Molesme (Côte-d'Or) fait actuellement l'objet d'une fouille "géoarchéologique »; il s'agit de dater et quantifier de façon aussi précise que possible les relations complexes entre les aménagements anthropiques et la dynamique alluviale. En effet, l'une des clefs de lecture du paysage historique réside en grande partie dans le décryptage de la réponse sédimentaire à la succession sur la longue durée, des modifications de l'environnement sous la double contrainte du climat et de l'action des sociétés humaines.

\section{LES PARAMÈTRES DU SYSTÈME ENVIRONNEMENTAL}

\section{LE FONCTIONNEMENT ACTUEL DU BASSIN VERSANT DE LA LAIGNE}

\section{LES CARACTÉRISTIQUES PHYSIQUES DU BASSIN VERSANT DE LA LAIGNE}

La Laigne est un affluent de rive gauche de la Seine (fig. 1). Son bassin versant, dont la superficie avoisine $590 \mathrm{~km}^{2}$, est délimité, au sud, par sa résurgence dans le bourg de Laignes (Côte-d'Or) et, au nord, par son point de confluence avec la Seine, à Bar-sur-Seine (Aube). Il se structure en trois unités morphologiques bien distinctes: les plateaux calcaires de la bordure sud-est du Bassin parisien, les versants marno-calcaires, la plaine alluviale parcourue par la rivière. L'impluvium est constitué principalement par les calcaires karstifiés de l'Oxfordien et du Kimméridgien qui culminent à environ $300 \mathrm{~m}$ d'altitude. Cette série domine les marnes oxfordiennes qui affleurent au pied de la cuesta du Châtillonnais, aux environs de Laignes. Encaissée d'une centaine de mètres dans ces plateaux, la vallée présente une largeur qui n'excède pas $500 \mathrm{~m}$. L'agglomération antique de Vertillum domine la vallée tandis que les principaux villages actuels, pour la plupart d'origine médiévale, en occupent les versants (Mangin, Mangin-Jouin, 1994; Berti et al., 1998) (fig. 2).

En l'absence de dépôts éoliens reconnus dans ce secteur du Bassin parisien, seuls les sols superficiels suivants ont été cartographiés: sols bruns argilo-caillouteux calcaires à calciques (rendisols à rendosols) sur les plateaux et sols bruns limono-argileux calcaires, rarement profonds (rendosols à calcosols), sur les pentes (Chrétien, 2000). Certains versants orientés à l'est et au sud-est présentent des accumulations cryoclastiques weichséliennes (grèzes litées) mises en place en contexte périglaciaire (Journaux, 1963; Loreau, Thierry, 1975).

Si les plateaux et les versants sont largement boisés (70\% de la surface du bassin versant), prairies et cultures composent le paysage actuel de la plaine alluviale (Berti et al., 1998).

$\mathrm{Au}$ milieu de la plaine alluviale, l'actuel chenal de la rivière, large d'une dizaine de mètres, trace de nombreux petits méandres. En périphérie des prairies alluviales, des zones marécageuses subsistent autour de la ferme de la Motte, ainsi qu'au lieu-dit l'Abîme, au nord de Molesme, en bordure de la plaine (fig. 2). L'essentiel du remplissage holocène de cette vallée est constitué de sédiments carbonatés d'origine chimique ou biochimique, épandages d'oncolites, de micrites blanchâtres ou de manchons calcaires et de planchers tuffeux comme c'est le cas pour de nombreuses rivières de climat tempéré qui drainent des bassins versants calcaires (Geurts, 1976; Adolphe, 1981, 1989; Chafetz, Folk, 1984; Freytet, 1989; Pedley, 1990; Freytet, Plet, 1996; Campy, Macaire, 2003, p. 279-282). Bien qu'ils soient généralement peu visibles du fait de leur enfouissement fréquent sous des limons détritiques récents, ces dépôts de précipitation représentent des volumes considérables. 


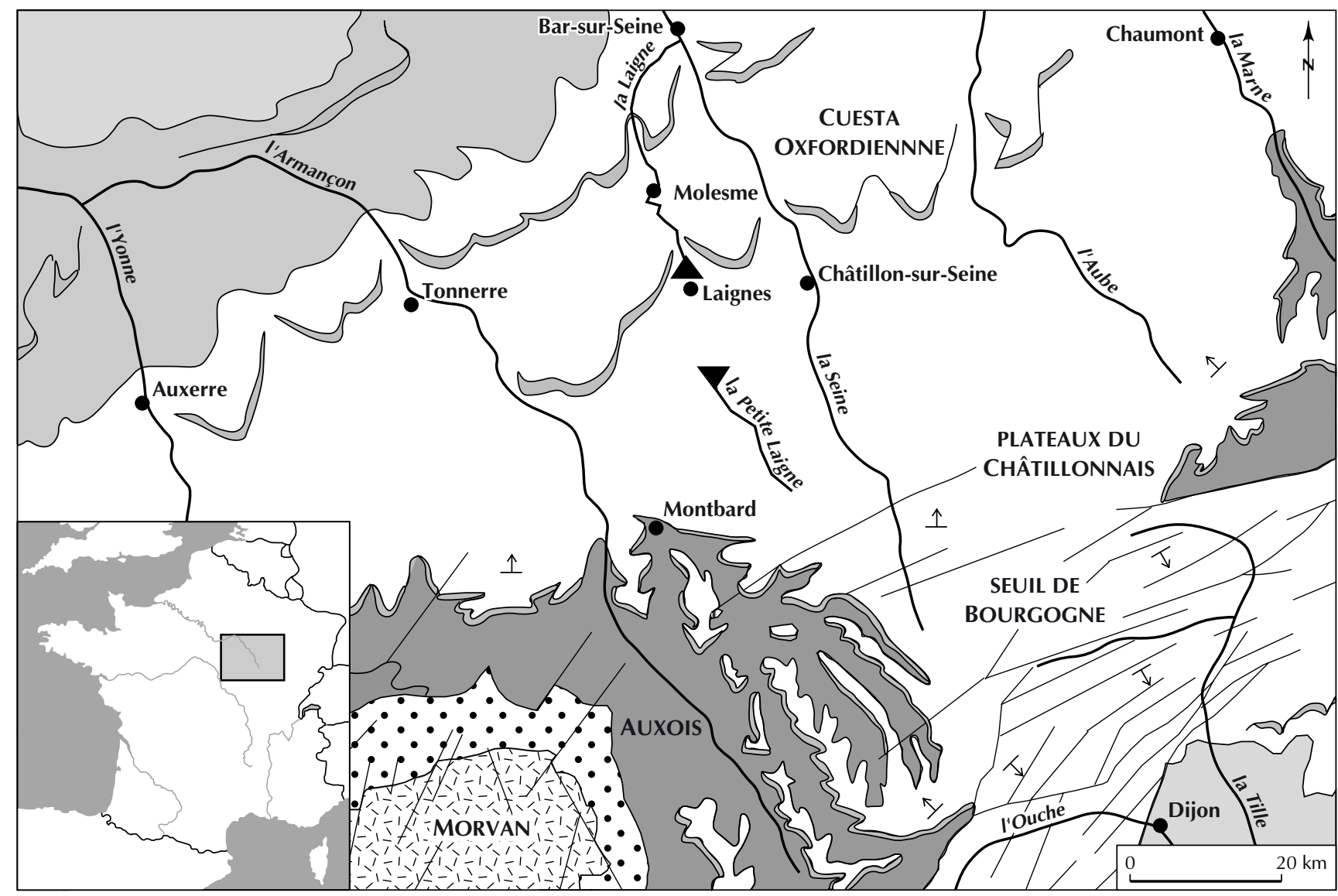

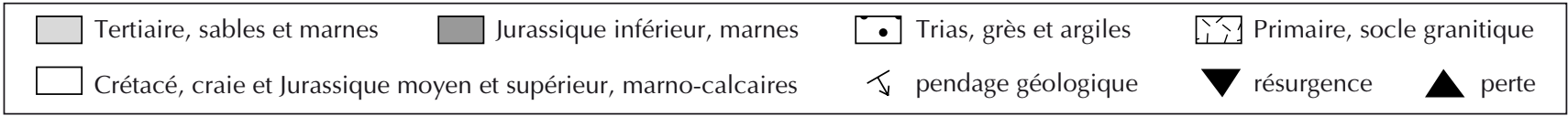

Fig. 1 - Le bassin versant de la Laigne en Bourgogne (DAO C. Petit, Université de Bourgogne).

\section{LE FONCTIONNEMENT HYDROLOGIQUE DE LA RIVIÈRE}

Le bassin hydrologique de la Laigne est soumis à un climat tempéré à tendance continentale; les précipitations moyennes annuelles s'élèvent à $800 \mathrm{~mm}$ pour 120 jours de pluie. Mai, juin et décembre sont les mois généralement les plus arrosés (Lenoir, Pagney, 1982). Le cours d'eau connaît un débit modeste: l'apport moyen annuel enregistré à la station hydrologique de Molesme est en effet de l'ordre de $3,5 \mathrm{~m}^{3} / \mathrm{s}$ et le débit d'étiage quinquennal tombe à $0,38 \mathrm{~m}^{3} / \mathrm{s}$ (Marion, 1982; Ingargiola et al., 1989) (fig. 3). Une part très importante de l'écoulement provient des sources karstiques: la plus importante d'entre elles, la résurgence de la Laigne, contribue à elle seule à $47 \%$ du débit moyen d'étiage. Les eaux présentent alors une teneur élevée en carbonate dissous qui peut précipiter. Après les hivers particulièrement pluvieux, les averses violentes provoquent d'importantes crues de printemps. Le débit instantané décennal atteint alors en moyenne les $29 \mathrm{~m}^{3} / \mathrm{s}$. À Molesme, ces crues décennales entraînent actuellement un débordement qui gagne toute la plaine d'inondation; elle sont suivies par de longues décrues qui s'étalent sur environ deux semaines.

\section{LES DONNÉES ARCHÉOLOGIQUES}

Attestée par l'archéologie, la photographie aérienne et les sources documentaires, l'occupation humaine du secteur paraît continue, au moins depuis la Protohistoire; les vestiges repérés sont répartis sur l'ensemble du bassin versant (Berti et al., 1998, p. 12 et 75) (pl. III hors texte). 


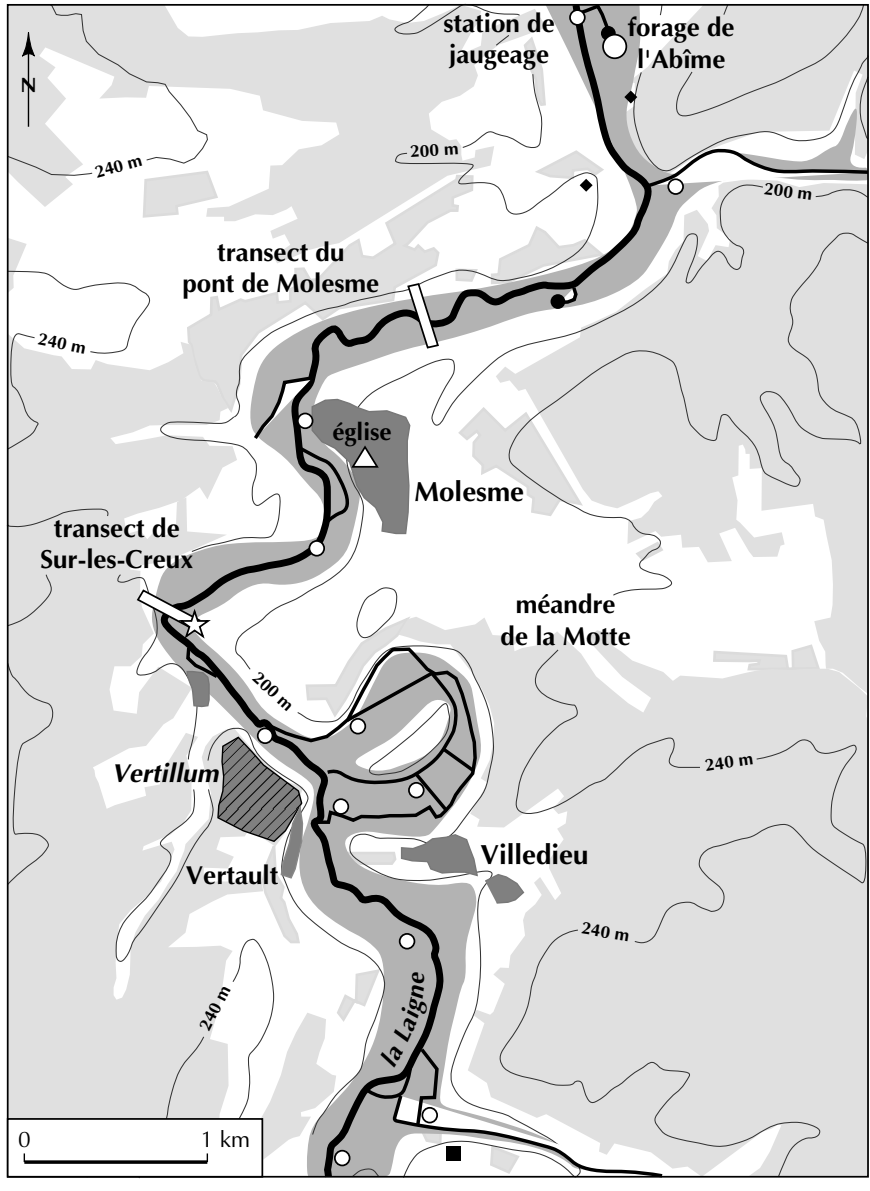

\begin{tabular}{|c|c|c|}
\hline village actuel & $\stackrel{s}{2}$ & site de Sur-les-Creux à Molesme \\
\hline tique & 0 & $\begin{array}{l}\text { forage palynologique du site de } \\
\text { I'Abîme à Molesme }\end{array}$ \\
\hline plaine d'inondation & O & forage géologique isolé \\
\hline forêts actuelles & & tranchées géologiques \\
\hline
\end{tabular}

Fig. 2 - Localisation des sondages et des transects géologiques dans la vallée de la Laigne, à proximité de l'agglomération gallo-romaine de Vertillum et de l'abbaye bénédictine de Molesme implantées sur les plateaux (DAO C. Petit, Université de Bourgogne).

\section{Pré- ET PRotohistoire ANCIENNE}

Les premiers témoignages de la présence humaine dans la vallée remontent au Paléolithique, mais ils restent rares: l'abri sous-roche de la Baume de Bâlot, au sud du bourg de Laignes, est occupé au Paléolithique moyen et à la fin du Paléolithique supérieur (Pautrat, 2002). Le Néolithique n’a laissé que peu de traces dans le pays. Il s'agit de mobilier

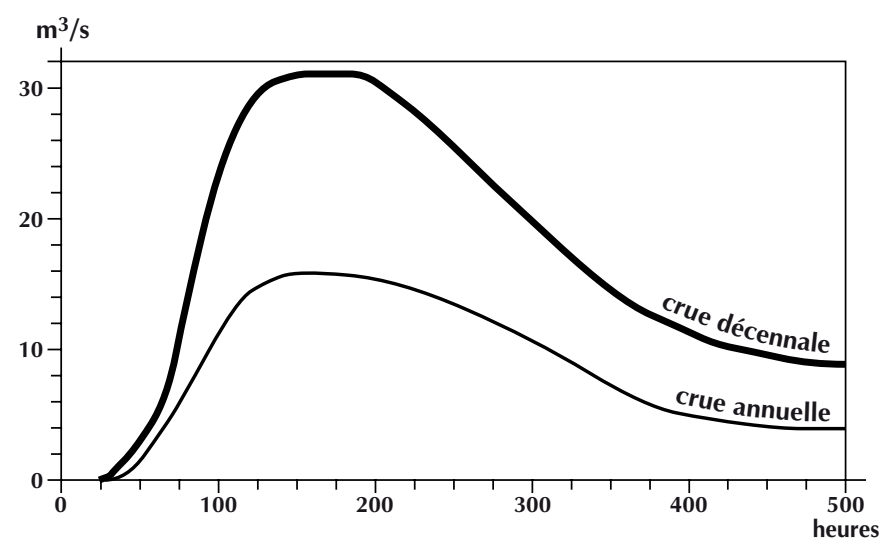

Fig. 3 - Hydrogrammes de crue de la Laigne établis à partir des enregistrements de la station de jaugeage située au nord de la commune de Molesme. Le pic de crue annuelle est atteint en 8 jours environ et la décrue s'étale sur plus de 2 semaines. Le débit d'une crue de récurrence décennale représente environ le double de celui d'une crue annuelle (DAO C. Petit, Université de Bourgogne).

lithique découvert sur les communes de Laignes, Nicey, Griselles et Molesme.

De l'âge du Bronze à La Tène moyenne, l'occupation du Châtillonnais se fait plus dense, mais c'est surtout dans la vallée de la Seine que se concentrent les témoignages archéologiques, essentiellement autour de la butte de Vix (Joffroy, 1960; Rolley, 2003). Dans la vallée de la Laigne, les indices archéologiques sont en revanche beaucoup plus rares; on retiendra en particulier les tumuli de Villedieu, les enclos circulaires de Marcennay et des Riceys, le mobilier lithique de Vertault, ainsi que le bracelet en or de Molesme (Berti et al., 1998). Cette faible densité d'occupation de la vallée de la Laigne tient probablement au fait que celle-ci se trouvait en marge de l'axe majeur d'occupation que constituait alors la trouée de la Seine (Maranski, 1997; Chaume, 2001).

\section{ÉPOQUES LATÉNIENNE ET GALLO-ROMAINE}

C'est à partir de La Tène finale que l'occupation de la vallée se fait nettement plus dense; elle semble s'organiser autour de deux pôles majeurs: le pied de la cuesta, avec les enclos carrés repérés en photographie aérienne sur Laignes et Griselles et le plateau, avec l'oppidum et les enclos de Vertault. C'est au pied de cet oppidum que se situe le site de Sur-les-Creux à Molesme. 
À l'époque gallo-romaine, l'agglomération secondaire de Vertillum succède à l'oppidum des Lingons (Mangin, Mangin-Jouin, 1994; Barral et al., 2002). Un important site rituel, du Haut-Empire, avec dépôts d'animaux sacrifiés a été découvert à l'extérieur du murus gallicus qui délimitait la ville (Jouin, Méniel, 2001).

Un réseau routier dense structure le territoire. L'axe majeur, de direction nord-sud, relie Alesia à Augustobona (Troyes, Aube) via Vertillum. Longeant la côte châtillonnaise d'est en ouest, une autre voie importante dessert les villes d'Agedincum (Sens, Yonne) et d'Endematunnum (Langres, Haute-Marne) ; elle passe à proximité de Laignes. À l'échelle locale, de nombreuses voies secondaires repérées en prospection pédestre ou aérienne dessinent un réseau centré sur l'agglomération de Vertillum.

Là aussi, deux pôles majeurs se distinguent, tous deux hérités de l'occupation laténienne:

- le premier est centré autour de l'agglomération de Vertillum (thermes, temple, forum, urbanisation en îlots) à proximité de laquelle de nombreuse petites villae exploitaient les terres des versants et des plateaux qui présentent des sols de qualité médiocre, mais légers. Les villages médiévaux forment une nouvelle trame de l'habitat en rupture avec la précédente même s'il existe des cas de continuité d'occupation entre villa et village: ainsi Pouilly (Poliacum), auquel succède Molesme, est certainement issu d'une villa antique. - le second pôle s'étend le long de la cuesta châtillonnaise: les villae de Nicey et de Lornay à Griselles ont été implantées sur les terres les plus riches de la région. Elles se caractérisent par leur grande taille et leur aspect luxueux; de nombreuses traces de parcellaire semblent se rattacher à ces établissements (Goguey, 2003).

\section{DU MOYEN ÂGE À NOS JOURS}

Le déclin urbain, amorcé dès la fin du III $^{\mathrm{e}}$ s., est attesté par l'abandon de l'oppidum de Vertillum (Vertault) à la fin du $\mathrm{V}^{\mathrm{e}} \mathrm{s}$. Deux nouveaux pôles périphériques se mettent alors en place, l'un au nord, aux Riceys dont la paroisse est mentionnée en 695 et l'autre au sud, à Laignes en 632; l'existence de nombreux villages est attestée avant la fin du XI ${ }^{\mathrm{e}} \mathrm{s}$. (Vertault en 1076, Villedieu en 1090). L'époque médiévale classique $\left(\mathrm{XI}^{\mathrm{e}}-\mathrm{XIV}^{\mathrm{e}} \mathrm{s}\right.$.) est celle d'une profonde transformation $\mathrm{du}$ paysage politique et religieux, et donc économique, de toute la vallée:

- l'abbaye bénédictine de Molesme, fondée en 1075 par saint Robert, gère, avec ses dépendances, une grande partie des terres cultivées et des bois. Les religieux implantent une série de fermes sur les rebords de plateaux, ainsi qu'en bas de versant;

- plus au sud, la ferme de la Motte, qui dépend de l'hôpital de Tonnerre fondé dès 1297, exploite le terroir autour du méandre fossile de Vertault-Villedieu (fig. 2);

- des pouvoirs féodaux laïcs se répartissent le reste du territoire (châteaux des Riceys, de Villedieu, de Griselles et de Laignes).

Parallèlement à cette gestion agricole, l'abbaye, l'hôpital de Tonnerre et la seigneurie de Villedieu mettent en place une exploitation systématique de la rivière: moulins, biefs, viviers et étangs jalonnent régulièrement le cours de la Laigne (Berti et al., 1998).

Dès le XVI ${ }^{\mathrm{e}} \mathrm{s}$, mais surtout au cours des XVII ${ }^{\mathrm{e}}-\mathrm{XVIII}{ }^{\mathrm{e}} \mathrm{s}$, les plateaux sont défrichés; des hameaux y sont implantés, bien que les ressources en eau y soient extrêmement rares.

L'exploitation des carrières de calcaire est florissante au XIX $^{\mathrm{e}}$ s. Créée à cette époque, la route départementale D 954 relie Laignes aux Riceys et recoupe le marais de la Motte qu'aucune voie ne traversait auparavant; c'est l'une des dernières grandes modifications du paysage de la vallée de la Laigne. Désormais, seule la révolution agricole actuelle menace ponctuellement cet héritage désormais situé à l'écart des principaux réseaux économiques. La lecture du paysage historique s'avère donc ici relativement aisée.

À l'échelle d'un petit bassin versant comme celui de la Laigne, dont la vallée focalise l'essentiel des implantations historiques, la fondation de Vertillum semble déterminante pour l'organisation de l'espace du bassin versant: les aménagements de cet espace socio-environnemental ont-ils eu une conséquence sur le milieu? (Van der Leeuw et al. dir., 2003). C'est le décryptage de la réponse sédimentaire qu'offre ce milieu anthropisé qui constitue l'objectif majeur de cette étude.

\section{L'ÉVOLUTION PALÉOENVIRONNEMENTALE DE LA VALLÉE}

Forages ponctuels, transects perpendiculaires à la vallée et fouilles ont constitué les trois niveaux d'investigation destinés à mettre en évidence les divers indices de transformation paléoenvironnementale: séquence palynologique des marais organiques bordant la vallée, coupes et forages sédimentologiques dans la plaine alluviale et étude géoarchéologique du site de Sur-les-Creux, implanté au bord de la rivière. 
$\begin{array}{lllllllllll}0 & 10 & 20 & 30 & 40 & 50 & 60 & 70 & 80 & 90 & 100 \%\end{array}$

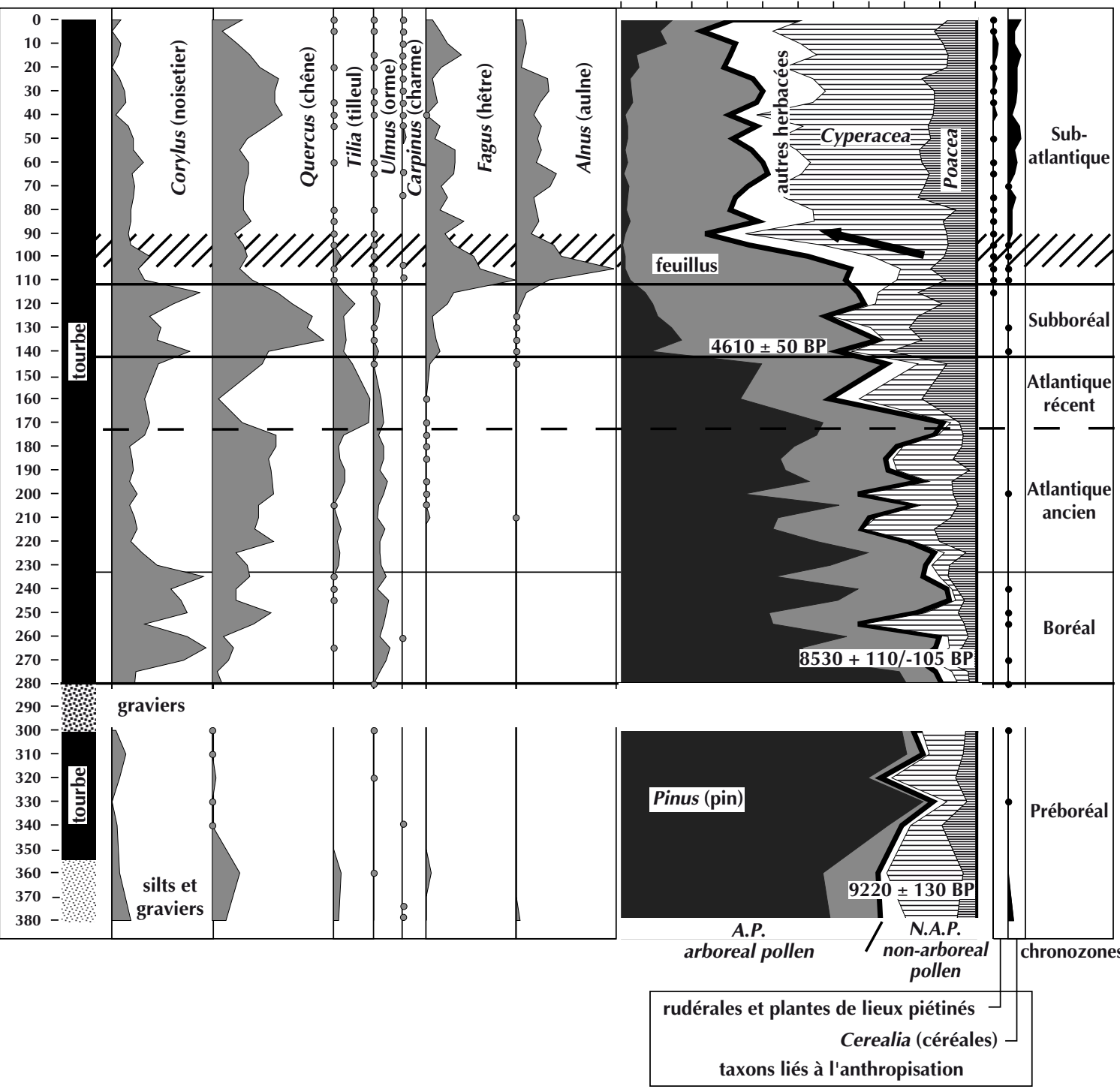

Fig. 4 - Diagramme palynologique simplifié du site de l'Abîme à Molesme (d'après Gauthier et al., 2006). Cette série tourbeuse s'est développée dans un milieux marécageux, à proximité d'une source, en bordure de vallée. Au cours du Subatlantique, la nette diminution des pollens d'arbres (flèche) et l'augmentation des taxons liés à l'anthropisation (zone hachurée) traduisent très probablement le début de l'exploitation agricole généralisée du bassin versant de la Laigne dans lequel s'implante l'agglomération antique de Vertillum (analyses E. Gauthier) (DAO E. Gauthier et C. Petit, Université de Bourgogne).

\section{LES INDICES PALYNOLOGIQUES D'ANTHROPISATION}

Parmi les nombreux forages carottés réalisés dans la plaine, la série tourbeuse la plus épaisse a été décelée au lieu-dit l'Abîme, en rive gauche de la Laigne. Un forage à la sonde russe a révélé une série essentiellement tourbeuse d'une épaisseur de près de $4 \mathrm{~m}$ qui a fait l'objet de prélèvements effectués tous les $5 \mathrm{~cm}$ (Gauthier et al., 2006) (fig. 4).
Les trois datations radiocarbone AMS réalisées sur des fragments ligneux du forage montrent l'ancienneté du remplissage et laissent à penser que l'essentiel de l'Holocène est ici représenté (tabl. I). Les informations paléoclimatiques issues de l'analyse palynologique sont conformes aux données habituelles caractérisant l'Holocène ${ }^{6}$ (Gauthier,

6. La chronologie de l'Holocène et la succession des évolutions culturelles comportent: le Préboréal (9200-8030 cal. BC), Mésolithique; le Boréal (8030-6900 cal. BC), Mésolithique pro parte et Néolithique 
Tabl. I - Liste des dates radiocarbone obtenues dans la vallée de la Laigne (calibration réalisée avec le programme de Stuiver et al., 1998).

\begin{tabular}{|c|c|c|c|c|}
\hline & Cote & Référence & Âge ${ }^{14} \mathrm{C} B P$ & $\begin{array}{c}\text { Calibration à } 2 \text { sigmas } \\
(95 \%) \text {, cal. BC }\end{array}$ \\
\hline \multirow{3}{*}{$\begin{array}{l}\text { Forage de } \\
\text { I'Abîme à } \\
\text { Molesme }\end{array}$} & 140-142 & AA-9139 & $4610 \pm 50 \mathrm{BP}$ & {$[3517,3121]$ cal. BC } \\
\hline & $268-270$ & AA-9140 & $8530 \pm 110 /-105 \mathrm{BP}$ & {$[7865,7349]$ cal. BC } \\
\hline & $367-369$ & AA-9141 & $9220 \pm 130 \mathrm{BP}$ & {$[8583,8225]$ cal. BC } \\
\hline \multirow{5}{*}{$\begin{array}{l}\text { Coupe du } \\
\text { pont de } \\
\text { Molesme }\end{array}$} & 130 & Ly-840 & $5130 \pm 120 \mathrm{BP}$ & {$[4250,3650]$ cal. BC } \\
\hline & 140 & Ly-839 & $5230 \pm 170 \mathrm{BP}$ & {$[4350,3700]$ cal. BC } \\
\hline & 205 & Ly-838 & $8720 \pm 150 \mathrm{BP}$ & {$[8250,7500]$ cal. BC } \\
\hline & 230 & Ly-837 & $9280 \pm 170 \mathrm{BP}$ & {$[9200,8200]$ cal. BC } \\
\hline & 250 & Ly-836 & $9900 \pm 290 \mathrm{BP}$ & {$[10700,8500]$ cal. BC } \\
\hline
\end{tabular}

2001). Laccent est donc mis ici sur les indices palynologiques d'anthropisation particulièrement originaux; en effet, contrairement à la plupart des analyses qui proviennent du Bassin parisien, de Lorraine, des Vosges, du Jura et des plaines de la Saône, aucun impact de l'homme sur la végétation proche de ce site n'est perceptible avant la transition Subboréal-Subatlantique, c'est-à-dire avant l'extrême fin de l'âge du Bronze. Il semble donc que les agriculteurs du Néolithique et de l'âge du Bronze ancien et moyen se soient totalement désintéressés de cette partie de la vallée. Ces données palynologiques confirment les premières conclusions issues des inventaires archéologiques.

C'est au cours du Subatlantique que le couvert forestier s'est transformé. La forêt, marquée par l'action de l'homme, est alors dominée par le chêne (Quercus) et le hêtre (Fagus); le noisetier (Corylus) ne cesse de baisser; l'orme (Ulmus), le tilleul (Tilia) et le frêne (Fraxinus) montrent des taux très faibles. Dans le premier tiers du Subatlantique, qui correspond approximativement à l'âge du Fer, l'impact de l'homme reste toutefois discret. Les indices polliniques d'anthropisation, grains de pollens de céréales et de plantains qui sont présents dès la cote $120 \mathrm{~cm}$, ne s'affirment véritablement qu'à partir de la cote $100 \mathrm{~cm}$, c'est-à-dire peu après le début du Subatlantique. Il faut en fait attendre la période correspondant à La Tène et à l'époque gallo-romaine pour que les indices deviennent plus évidents; ils sont certainement contemporains de l'installation de l'oppidum de Vertillum. S'ensuit un léger fléchissement des indices polliniques d'anthropisation, en particulier la présence moins

pro parte; l'Atlantique (6900-3450 cal. BC), Néolithique pro parte; le Subboréal (3450-820 cal. BC), Néolithique et âge du Bronze; le Subatlantique (820 cal. BC à aujourd'hui), de l'âge du Fer à aujourd'hui (Magny, 1995). constante de céréales, ce qui pourrait correspondre à une légère déprise agricole, principalement centrée sur le haut Moyen Âge. Enfin, le développement du chêne apparaît comme la probable conséquence de la gestion monastique des forêts de Molesme.

\section{LA SÉDIMENTATION ALLUVIALE HOLOCÈNE AUTOGHTONE PUIS DÉTRITIQUE}

\section{LES UNITÉS STRATIGRAPHIQUES ALLUVIALES}

Dans la zone axiale de la vallée, des coupes naturelles ainsi qu'une vingtaine de sondages carottés ont permis d'identifier trois unités stratigraphiques superposées. La géométrie, les âges et les principaux faciès de ces trois unités ont été reconnus sur les deux transects effectués perpendiculairement à l'axe de la vallée (pl. IV hors texte):

- le premier a été réalisé dans un secteur étroit de la vallée, au niveau du pont de Molesme; il passe par une coupe datée par ${ }^{14} \mathrm{C}$ (Puisségur, 1976) (tabl. I et pl. V hors texte);

- le second prolonge, sur la rive gauche de la rivière, la fouille géoarchéologique du site de Sur-les-Creux, dans une partie plus large de la vallée.

Les unités suivantes se lisent de bas en haut:

Unité 1. Incisé dans les calcaires oxfordiens, le fond de la vallée est occupé, sur une épaisseur maximale de $3 \mathrm{~m}$, par des graviers et des galets constitués de faciès calcaires variés. $\mathrm{Au}$ pont de Molesme, la malacofaune présente de fortes proportions d'espèces steppiques. Ces dépôts graveleux sont caractéristiques d'un système fluviatile en tresses, mis en place sous un climat périglaciaire au cours du WeichsélienTardiglaciaire.

Unité 2. La deuxième unité, dont l'épaisseur avoisine les $3 \mathrm{~m}$, est majoritairement formée de dépôts principalement carbonatés ou organiques. Elle débute au Préboréal et se clôt brutalement au début du Subatlantique. Dans les zones les plus étroites de la vallée, les barres oncolitiques sont plus importantes; en revanche, les niveaux organiques sont plus développés en bordure et dans les zones plus larges. L'analyse malacologique montre bien, du fait de la présence d'une faune terrestre à affinités forestières, que les niveaux organiques qui se suivent d'un forage à l'autre correspondent à des phases d'assèchement très probablement d'origine climatique qui affectent l'ensemble de la vallée. Ces phases modifient le bilan hydrologique de la rivière, mais ne semblent pas avoir eu d'incidence sur la quantité d'apports sédimentaires issus des versants. Bien qu'originales du fait de la prépondérance des processus 
de précipitation carbonatés, ces séquences holocènes sont caractéristiques des petits bassins versants essentiellement calcaires du Bassin parisien (vallée de la Beuvronne) (Pastre et al., 2002; Orth et al., 2004) ou du Tournugeois (Freytet, 1989; Freytet, Plet, 1996).

Unité 3. La troisième et dernière unité est majoritairement constituée de «limons gris » qui recouvrent l'essentiel de la plaine d'inondation actuelle et ce sur une épaisseur moyenne de $50 \mathrm{~cm}$, qui peut atteindre $80 \mathrm{~cm}$ par endroits. Le matériel archéologique contenu à la base n'est jamais antérieur au $\mathrm{I}^{\mathrm{er}} \mathrm{s}$. L'homogénéité de ces limons carbonatés est caractéristique des sols alluviaux intensément bioturbés par les lombrics (Courty et al., 1989; Berger, 1995). Ces limons détritiques se mettent en place dans une plaine alluviale inondable, au sein d'un système fluviatile du type " système méandrisant à chenal unique ». Les cortèges de malacofaune mixte, aquatique et terrestre, témoignent de la récurrence des inondations dans la plaine alluviale. La malacofaune terrestre est caractéristique des milieux extrêmement ouverts, ce qui tranche radicalement avec celle des unités antérieures, riches en espèces forestières et semi-forestières. Ce changement radical de la sédimentation entre les unités 2 et 3 s'avère contemporain d'une ouverture du milieu: la forêt alluviale laisse la place à des prairies herbacées; l'absence de gastéropodes marqueurs d'une dégradation climatique confirme l'hypothèse d'un contrôle anthropique de cette modification environnementale. Ces interprétations sont tout à fait conformes à celle que proposent S. Martin et F. Magnin (2002) au sujet de la plaine alluviale de l'Ubac (Vaucluse) soumise à un fort développement de l'occupation anthropique entraînant une ouverture du milieu au cours de La Tène finale et de l'époque gallo-romaine.

\section{LE DÉCLIN DE LA SÉDIMENTATION CARBONATÉE AU DÉBUT DU SUBATLANTIQUE}

Dans la vallée de la Laigne, le déclin rapide de la production carbonatée au début du Subatlantique ne peut pas s'expliquer par le seul facteur climatique, bien mis en évidence dans d'autres études (Weisrock, 1986; Lécolle, Létolle, 1989; Magny, 1995; Van Geel, Magny, 2002). La nature essentiellement calcaire du bassin versant de la Laigne induit une sédimentation préboréale-subboréale de la plaine alluviale principalement constituée de sédiments autochtones, de nature organique (tourbes et sols noirs marécageux) ou carbonatée (barres de concrétions et travertins résultant de la précipitation des carbonates en solution dans l'eau de la rivière). La présence de sédiments argileux et limoneux subatlantiques traduit des apports détritiques mis en place par ruissellement le long des versants, apports redistribués par le cours d'eau dans la plaine alluviale. L'abondance du matériel détritique témoigne donc de la forte érosion des sols des plateaux et des versants alors mis en culture (Bichet et al., 1997). Ce contrôle anthropique de la sédimentation carbonatée a déjà été démontré dans d'autres sites alluviaux ou lacustres (Vaudour, 1986; Bichet et al., 2002) ; son intensité peut être abordée par l'étude des coupes géoarchéologiques effectuées sur le site de Sur-lesCreux.

\section{LE SITE DE SUR-LES-CREUX À MOLESME}

L'ouverture en 1996 du chantier archéologique de Molesme a été l'occasion de mettre en œuvre et de tester une démarche innovante que nous pouvons qualifier de "géoarchéologique », puisqu'elle associe étroitement et combine sur un même site les prospections géophysiques, l'ouverture de grandes tranchées géologiques destinées à replacer le site archéologique dans son environnement proche et enfin la fouille d'aménagement antique de paléochenaux ou de structures (fossés et bassin), éléments majeurs d'un vaste site archéologique en cours de fouille (fig. 5 et 6). La combinaison de ces trois démarches permet de proposer une reconstitution des transformations tant sédimentaires qu'anthropiques de ce secteur de la plaine alluviale.

\section{LES DONNÉES ARCHÉOLOGIQUES}

Le chantier concerne un site inscrit dans la boucle d'un méandre de la Laigne. La photographie aérienne prise par René Goguey lors de la grande sécheresse de 1976 et les prospections pédestres laissaient penser qu'il s'agissait là d'une importante installation gallo-romaine de bord de rivière, édifiée en relation avec l'agglomération toute proche de Vertillum; l'hypothèse de la présence d'installations portuaires avait même été émise (Mangin, Mangin-Jouin, 1994). Le choix d'implantation d'une fouille géoarchéologique semblait alors se justifier par le fait que de tels aménagements avaient nécessairement généré des modifications voire des bouleversements du cours d'eau, tant de son tracé que de son régime. Les structures archéologiques ont en effet été implantées en rive droite de la rivière, tout en bas de la pente douce du versant oriental de la vallée; ces installations occupent la quasi-totalité de 


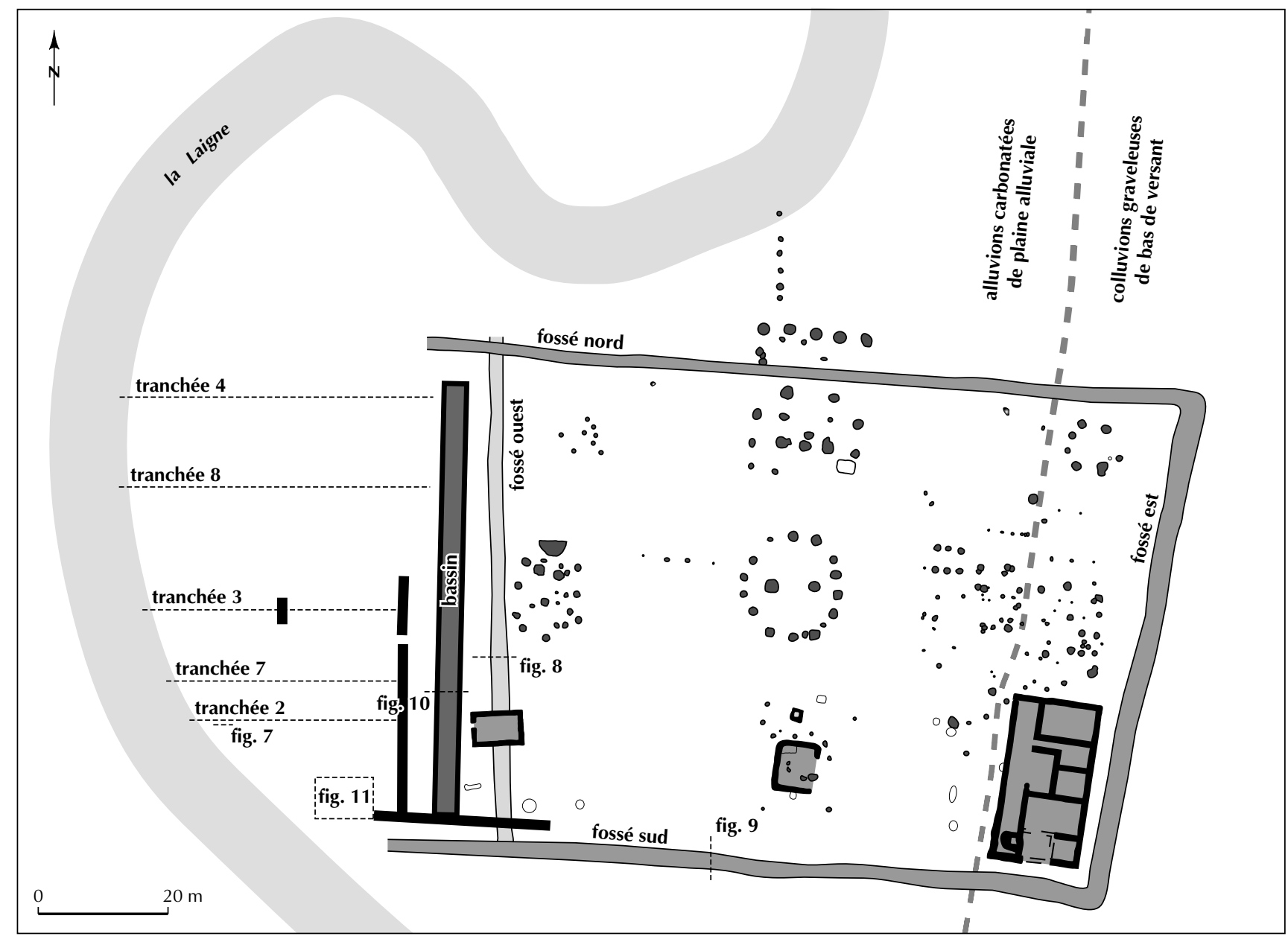

- trou de poteau $\bigcirc$ fosse $\bigsqcup$ fossé laténien $\square$ fossé gallo-romain

Fig. 5 - Plan schématique des principales structures archéologiques du site de Sur-les-Creux à Molesme. Les tranchées géoarchéologiques ainsi que les principales illustrations de la fouille ont été positionnées (DAO C. Petit, Université de Bourgogne).

Fig. 6 - Le site de Sur-les-Creux à Molesme en 2001: à droite, le grand bassin occupant toute la longueur du méandre et, à gauche, le bâtiment maçonné antique au pied de versant (cliché C. Petit, Université de Bourgogne).

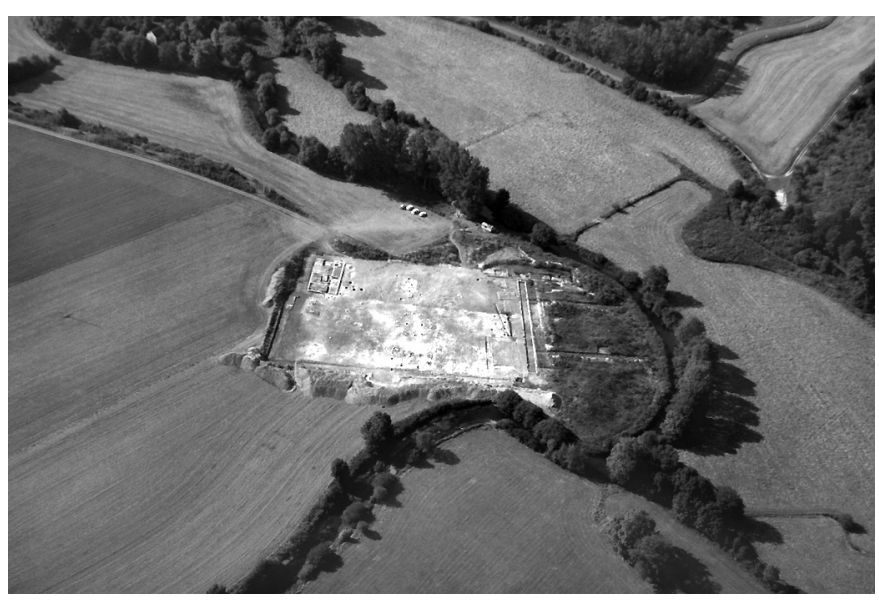


l'espace délimité par le méandre prononcé que dessine la Laigne à cet endroit. Immédiatement en amont, l'agglomération antique de Vertillum, bâtie sur l'éperon, domine le site de Molesme.

La fouille a permis de mettre en évidence deux phases continues d'occupation du site ${ }^{7}$ :

- la première est matérialisée par des fossés et des bâtiments sur poteaux de bois datés de La Tène finale ${ }^{8}$;

- la seconde, datée de la période gallo-romaine précoce, se met en place à l'époque augustéenne; elle est constituée de deux ensembles de structures maçonnées: à l'est, les bâtiments découverts ont été édifiés dans l'espace limité par l'enclos protohistorique; en revanche, à l'ouest, un grand bassin piscicole a été creusé dans la zone délimitée par le fossé ouest de l'enclos laténien et le bord de la rivière; il est accompagné d'un petit bâtiment rectangulaire et de murs de clôture installés sur un épais radier de pierres destiné à stabiliser les terrains inondables du fond de la vallée. L'occupation gallo-romaine s'achève autour de 60-70 apr. J.-C. 9

L'interprétation complète du site archéologique dont la fouille s'est achevée fin 2004 reste encore à préciser. Abandonnant progressivement l'interprétation portuaire puis agricole de ce site, l'économie générale du site (l'organisation des bâtiments en bois, la présence de nombreuses amphores Dressel 1, l'organisation des rejets de faune, et finalement la découverte d'une statue-bloc de facture celtique) nous engage à interpréter ce site comme un sanctuaire celtique, puis gallo-romain, qui reste original à ce jour (Petit, Wahlen, 2002, 2004).

\section{L'APPROCHE SPATIALE}

La photographie aérienne infrarouge prise par René Goguey en 1976 a été redressée à l'aide du logiciel Airphoto. L'image obtenue permet désormais de géoréférencer les structures archéologiques ou fluviales, en particulier les paléochenaux de la rivière et des anomalies dont l'interprétation immédiate s'avère délicate (pl. VI, n $\mathrm{n}^{\mathrm{O}} 1$ hors texte). Il en est ainsi de la trace claire, de tracé courbe

7. Le mobilier céramique a été étudié par P. Barral, M. Joly, F. Olmer et A. Delor, les monnaies par L. Popovitch, le mobilier métallique par J.-P. Guillaumet et le mobilier osseux par P. Méniel. Les datations dendrochronologiques ont été revues par O. Girarclos (Cèdre, Besançon).

8. L'un des poteaux retrouvé en place a fourni une date d'abattage postérieure aux années 60 av. J.-C.

9. Aucune production céramique d'époque flavienne ou postérieure n'a été découverte sur le site en stratigraphie. et quasi-parallèle au tracé de la Laigne actuelle; elle a fait l'objet d'investigations géophysiques et géologiques complémentaires.

La cartographie de la résistivité électrique ${ }^{10}$ apparente des terrains superficiels a été réalisée dans le méandre de la Laigne avec un dispositif de prospection du type «pôlepôle ".

Le secteur cartographié se découpe en deux zones de résistivité distinctes (pl. VI, no 2 hors texte) :

- la partie occidentale apparaît plus conductrice. La structure rectiligne résistante correspond à l'anomalie repérée sur les clichés: il s'agit en fait d'une importante barre sableuse comme l'ont ensuite révélé les coupes géoarchéologiques effectuées sur le terrain (pl. VII hors texte);

- la partie orientale correspond à la zone archéologique proprement dite; elle présente des anomalies qui apparaissent essentiellement résistantes parce que liées à la présence, soit de murs, soit de structures en creux (bassins, fossés, etc.) dont le remblaiement est principalement constitué de pierres. Mais en tout état de cause, l'identification et la datation de l'ensemble des indices photographiques et des réponses géophysiques auraient été difficiles en l'absence de fouilles.

\section{LA STRATIGRAPHIE DES TRANCHÉES GÉOARCHÉOLOGIQUES}

La douzaine de grandes tranchées réalisées du site jusqu'à la berge de la Laigne donne désormais une vision précise de l'évolution de la sédimentation alluviale aux cours des périodes historiques. Les cinq coupes parallèles permettent de suivre la géométrie des unités sédimentaires entre le site archéologique stricto sensu et la rivière actuelle (pl. VII hors texte); les autres complètent le dispositif

10. La résistivité électrique (en ohm x mètre) caractérise la capacité du terrain à s'opposer à la propagation du courant électrique. La méthode de mesure de la résistivité électrique du sol en courant continu utilise quatre électrodes. Deux d'entre elles sont des électrodes d'injection de courant, les deux autres permettent de mesurer la différence de potentiel pendant l'injection du courant. L'un des dispositifs les plus simples utilise deux électrodes mobiles, l'une de courant et l'autre de potentiel, associées à deux électrodes fixes positionnées loin des précédentes («à l'infini »). Ce dispositif, appelé "pôle-pôle » est donc simple à mettre en œuvre sur un terrain dégagé. On déduit des mesures effectuées (valeur du courant injecté et différence de potentiel mesurée) une résistivité électrique apparente en tenant compte de la disposition géométrique des électrodes. Dans le cas de ce dispositif, la profondeur d'nvestigation moyenne est voisine de la distance entre les électrodes proches, soit $1 \mathrm{~m}$ dans notre cas. 


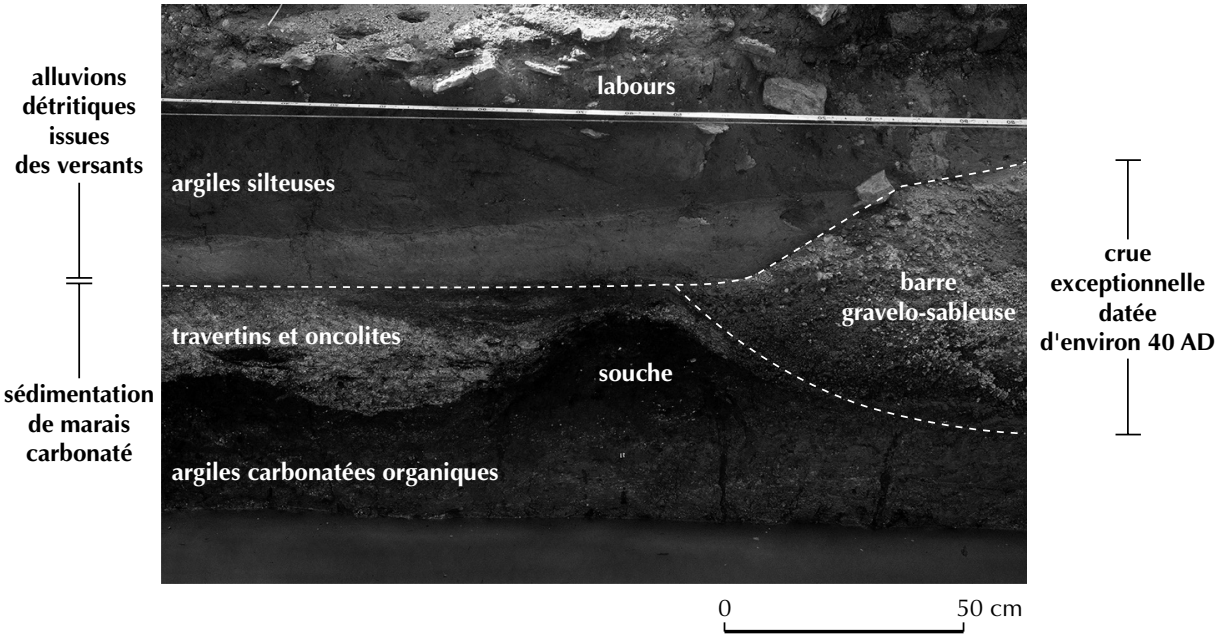

Fig. 7 - Détail de la bordure occidentale de la barre gravelo-sableuse de crue de la Laigne; cet événement exceptionnel se place stratigraphiquement au moment où la plaine marécageuse est colmatée par les apports détritiques issus des versants dans la première moitié du $I^{e r}$ s. apr. J.-C. La photo a été prise dans la tranchée 2 (cliché C. Petit, Université de Bourgogne). d'investigation, afin de pouvoir réaliser une reconstitution tridimensionnelle du méandre de la Laigne. Les faciès sédimentaires, leur géométrie et leurs milieux de dépôt sont ici présentés de bas en haut.

\section{LES ARGILES CARBONATÉES ET/OU ORGANIQUES}

Cette série, correspondant à l'unité 2 de la plaine alluviale (pl. IV hors texte), présente une grande extension spatiale, mais montre de fréquents changements de faciès (barres oncolitiques, débris organiques accumulés en périphérie...) qui, cependant, témoignent tous d'un milieu essentiellement marécageux (Brown, 1997, p. 51). Elle constitue le substrat du site affleurant sous les labours dans la zone d'implantation de l'enclos protohistorique; à l'ouest du bassin gallo-romain, à proximité du lit mineur de la rivière, elle a été repérée en fond de tranchée, à une profondeur qui atteint les $80 \mathrm{~cm}$. Cette différence topographique met ainsi en évidence une incision pratiquée par la rivière. Cet événement que l'on peut attribuer à l'époque subboréale ménage ainsi à l'est, en bordure de vallée, une terrasse alluviale qu'occupe ensuite l'enclos laténien.

\section{LES SABLES ET GRAVIERS DE LA BARRE DE GRUE}

Les faciès sableux repérés en fond de chenal et sur les berges du chenal de la Laigne renvoient au fonctionnement classique d'une rivière méandrisante; en revanche, la barre sablo-graveleuse correspondant à l'anomalie repérée sur la photographie aérienne et la carte géophysique résulte d'un accident hydrologique majeur, identifié et daté par la fouille (fig. 7). Longue d'environ $30 \mathrm{~m}$ et d'une largeur maximale de $9 \mathrm{~m}$, cette barre est conservée sur $2 \mathrm{~m}$ d'épaisseur. Du mobilier archéologique y a été découvert, constitué de fragments remaniés d'amphores et de tessons de céramiques augustéennes peu roulés. Plusieurs caractères sédimentologiques distinguent ce corps sédimentaire d'une simple barre progradante de méandre et l'identifient à un dépôt événementiel de type catastrophique mis en place lors d'une crue unique, d'une ampleur exceptionnelle:

- cette barre graveleuse est dans son ensemble très homogène et d'une granulométrie gravelo-sableuse inhabituelle dans cette plaine alluviale;

- elle se présente comme un corps discontinu, sans aucun passage latéral de faciès avec les dépôts avoisinants;

- elle occupe l'un des chenaux de la rivière (chenal B) et formait certainement à l'origine un relief dépassant de plus de $1 \mathrm{~m}$ le niveau moyen de la plaine; la mise en place de cette barre suppose la présence de courants localement violents, ainsi que d'un niveau d'eau très élevé dans la plaine;

- les moellons découverts à la base de cette barre témoignent très certainement de la destruction de maçonneries situées à quelques mètres en amont; peut-être s'agissait-il d'aménagements de berges?

Cette barre de crue peut être datée de 40 apr. J.-C. avec une incertitude de l'ordre de 20 ans, car sa mise en place s'intercale entre deux événements archéologiques bien datés dans le chenal A (pl. VII hors texte). 


\section{LES LIMONS DE DÉBORDEMENT}

Constitués de particules de la taille des silts et d'argiles mis en place par décantation lors des crues, ils correspondent aux dépôts les plus récents (unité 3 de la plaine alluviale) et colmatent les reliefs antérieurs. Leur épaisseur varie de $0 \mathrm{~m}$ à $1,80 \mathrm{~m}$ en bordure de la Laigne actuelle, mais reste le plus souvent voisine de $0,60 \mathrm{~m}$. Les niveaux de destruction du site archéologique et les labours récents scellent ou remanient ces dépôts limoneux.

La géométrie des unités alluviales est désormais bien cernée, non seulement à l'échelle de la plaine mais aussi à celle du méandre de Molesme, mais seule la fouille des structures archéologiques (fossés, bassin piscicole, aménagements de chenaux) permet de dater précisément les changements qui affectent les processus alluviaux au début de l'Antiquité.

\section{LES STRUCTURES ARCHÉOLOGIQUES}

\section{LES FOSSÉS}

L'enclos mis en place à l'époque laténienne a été partiellement fouillé par une série de sondages systématiques espacés de $7 \mathrm{~m}$. La reconstitution détaillée de l'alluvionnement comblant ces structures excavées et la chronologie des apports anthropiques volontaires de comblement (rejets de matériaux de construction) ont été mis en évidence par la fouille de l'angle sud-ouest de l'enclos, situé au plus près de la rivière (fig. 5 ). L'analyse des coupes conduit à distinguer de façon très nette deux réalités structurelles et chronologiques. Le remplissage du fossé occidental est conservé dans son état laténien, scellé par les radiers galloromains qui entourent le bassin piscicole. En revanche, l'état du fossé sud résulte d'un recreusement et d'une prolongation de l'enclos en direction de la rivière lors de l'installation du bassin piscicole à l'époque gallo-romaine. Il est très probable que ce fossé, comme ceux du nord et de l'est, soit d'origine protohistorique, mais ce premier état n'a pas été formellement reconnu.

\section{Le fossé laténien}

D'une profondeur de près de $1 \mathrm{~m}$ et d'une largeur d'environ $2 \mathrm{~m}$, le fossé présente un profil évasé et un fond relativement plat (fig. 5 et 8 ). La majeure partie du remplissage, principalement constituée d'argiles carbonatées et de sables, est d'origine alluviale. Riche en matières orga-
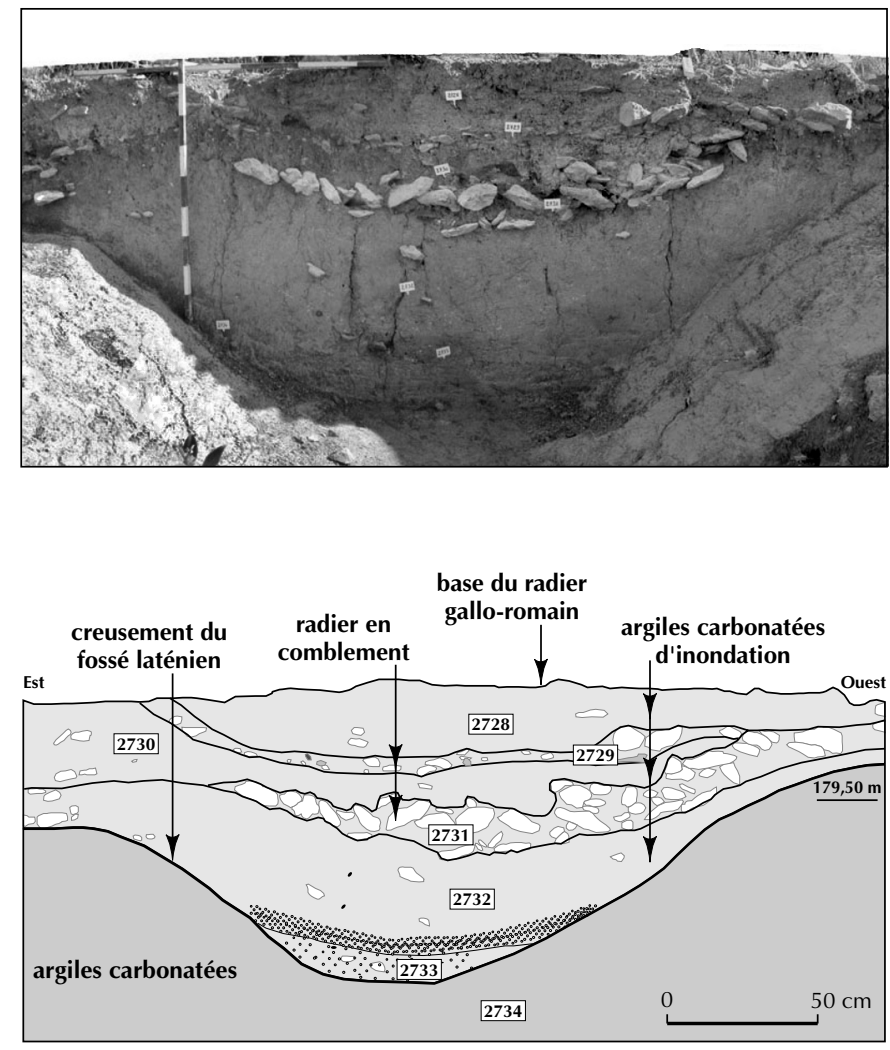

Fig. 8 - Coupe du fossé ouest laténien (cliché et DAO C. Petit, Université de Bourgogne).

niques, le fin liseré d'argiles sombres qui tapisse le fond témoigne de l'état marécageux de ce fossé, creusé sous le niveau de la nappe phréatique permanente. Par la suite, une série de dépôts d'argiles à concrétions carbonatées, de $10 \mathrm{~cm}$ à $40 \mathrm{~cm}$ d'épaisseur, intercalée avec des apports volontaires de pierres, se serait mise en place lors des phases d'inondation. Ces niveaux sont pauvres en mobilier céramique, qui se résume à quelques tessons mêlés à des ossements. Cependant, la présence dans ces sédiments de charbons épars provenant du lessivage du site indique que les inondations étaient régulières à l'époque laténienne. De plus, quelques fins dépôts de sables bien triés, de quelques centimètres de puissance, constituent le témoignage sédimentaire de crues de grande amplitude et de récurrence, peut-être centenale.

\section{Les fossés gallo-romains}

Identifié dans les autres fossés de l'enclos, en particulier le fossé sud qui se prolonge vers la rivière, un second 
creusement recoupe le comblement du fossé occidental (fig. 5 et 9). Le remplissage de ce deuxième creusement est, dans un premier temps, composé de pierres et de moellons mis en place depuis l'intérieur de l'enclos, par débordement latéral lors de l'installation des radiers antiques. Dans une seconde phase, des limons gris-jaunes comblent le fossé sud. Très épais par endroits, quasiment dépourvus de mobilier, ces limons détritiques témoignent d'un alluvionnement rapide qui comble ce fossé au cours de l'occupation antique du site. Au sommet du remplissage limoneux, un recreusement décalé vers l'extérieur de l'enclos recoupe les niveaux sous-jacents. Il est peu prononcé et se trouve comblé de matériaux de constructions; le mobilier céramique épars permet de dater cet épisode de l'époque julioclaudienne.

L'analyse des fossés met donc bien en évidence le fait que ce sont les structures archéologiques excavées qui ont enregistré la chronique hydrologique, avec les crues annuelles à décennales, ainsi que le changement radical de la nature des apports sédimentaires, carbonatés à l'époque laténienne, puis détritiques dès le Haut-Empire. L'examen du remplissage du grand bassin antique confirme ces observations.

\section{LE BASSIN PISCICOLE}

\section{Les données archéologiques}

Le grand bassin maçonné, de 63,80 m de long sur 3,20 m de large, occupe toute la longueur du méandre actuel de la Laigne. Son identification comme vivier piscicole repose sur ses caractéristiques techniques rapportées aux traités agricoles antiques, en particulier le De re rustica écrit par Columelle au I ${ }^{\mathrm{er}}$ s. (Zeepvat, 1988; Petit et al., 2005). Ce bassin, dont la hauteur conservée n'excède pas $0,80 \mathrm{~m}$, est simplement excavé dans les argiles alluviales imperméables. Son fond, quasiment horizontal, n'est pas dallé et ne présente pas de bonde; il ne pouvait donc pas se vidanger par le fond. En amont, le sommet du mur sud présente en son centre une échancrure rectangulaire correspondant à l'extrémité d'une conduite d'amenée d'eau. La conservation médiocre de l'exutoire ne permet pas de reconstituer les détails de son dispositif; il est certain cependant qu'il fonctionnait sur le principe du trop-plein, par évacuation des eaux en aval dans la rivière. À la périphérie immédiate du bassin, de larges surfaces empierrées ont été aménagées qui, stabilisant les sols alluviaux, permettaient de circuler et de travailler. C'est dans cette zone qu'un petit local maçonné $(7,20 \mathrm{~m} \times 5,10 \mathrm{~m})$, dont la fonction exacte n'est pas encore
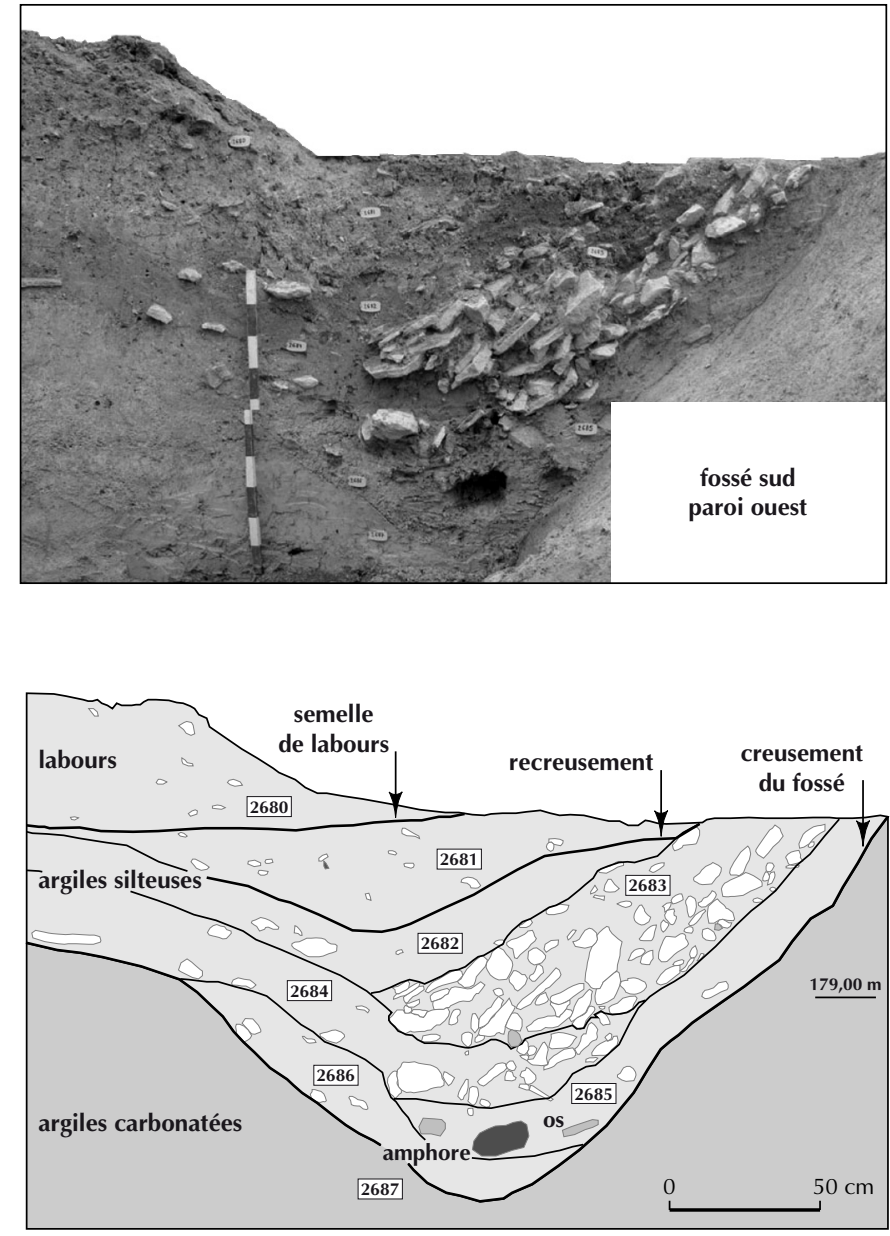

Fig. 9 - Coupe du fossé sud gallo-romain (cliché et DAO C. Petit, Université de Bourgogne).

établie, a été mis au jour. Cependant, la présence dans les fondations d'un lot de six monnaies romaines, trois deniers républicains, un as et deux quadrans augusto-tibériens, permet de dater cette installation du début du $\mathrm{I}^{\mathrm{er}} \mathrm{s}$. Sa durée de fonctionnement apparaît de façon étonnante assez courte puisque, bâti vers les années 20 apr. J.-C., le bassin paraît avoir été brusquement abandonné, puis immédiatement comblé avant la période flavienne, soit aux alentours des années 60 apr. J.-C. Cette datation repose en partie sur l'étude de la céramique sigillée, particulièrement celle issue du comblement final du bassin.

\section{LA STRATIGRAPHIE DU REMPLISSAGE}

L'examen de ce comblement permet d'établir la stratigraphie suivante (fig. 10) : 

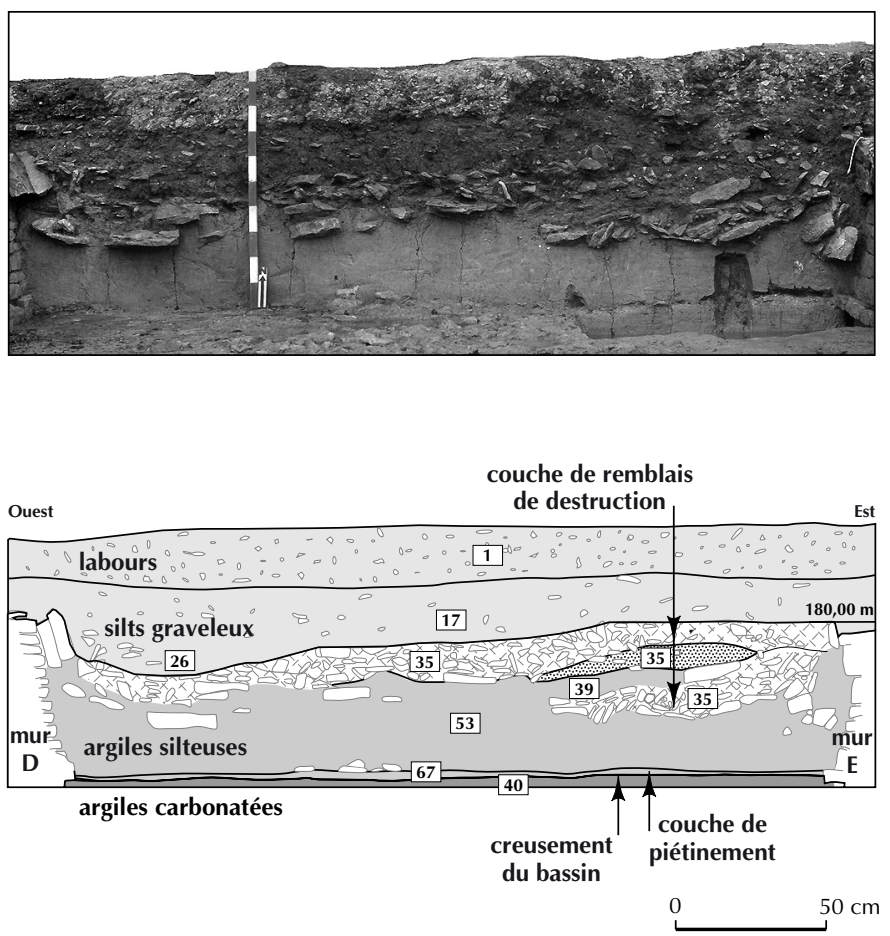

Fig. 10 - Coupe du remplissage du bassin (cliché et DAO C. Petit, Université de Bourgogne).

- quasi horizontal, le fond du bassin est matérialisé par un niveau de petits cailloux et de sables mêlés à une matrice argileuse, le tout reposant sur le substrat géologique excavé. Aucun mobilier ni écorestes (arêtes, écailles, graines...) n'y ont été récoltés, ce qui laisse à penser que le bassin était régulièrement nettoyé;

- une couche de silt argileux, homogène et d'une épaisseur d'environ $40 \mathrm{~cm}$, comble le bassin sur toute sa longueur. Il s'agit de limons d'inondation issus des débordements de la rivière intervenus lors de la phase d'abandon de la structure. L'analyse pétrographique et micromorphologique de ces sédiments très bioturbés montre qu'ils présentent les mêmes caractéristiques que ceux du comblement des fossés gallo-romains.

- la suite du remplissage est constituée par des déversements de blocs, pierres et rares moellons résultant de la destruction de bâtiments maçonnés situés plus à l'est. La partie sud du remplissage se distingue par la présence, au même niveau que les épandages de pierres en question, de moellons tombés en rang, face contre terre; il s'agit d'un pan effondré du mur G qui clôt le site immédiatement au sud. Le volume de ces matériaux d'inondation retenus par le bassin, soit environ $80 \mathrm{~m}^{3}$, correspond à un taux de piégeage considérable, de près de $1 \mathrm{~cm} / \mathrm{an}$, sur une durée qui n'excède pas une quarantaine d'années.

\section{L'AMÉNAGEMENT D'UN CHENAL ANTIQUE}

L'un des chenaux situé dans la partie occidentale du site, au-delà du bassin piscicole, a été partiellement fouillé (chenal A, pl. VII hors texte). Le fond était barré par un dispositif en bois dont subsistait une grande poutre de 6,50 m de long, aux extrémités ancrées dans les deux berges (fig. 11). Lanalyse dendrochronologique de cette pièce de chêne renvoie à une date d'abattage située aux environs de 20 apr. J.-C. La fonction exacte de ce dispositif reste à déterminer avec précision, mais sa position stratigraphique tant vis-à-vis de la rivière que des structures maçonnées antiques apporte de nombreux renseignements. Le mur G, qui clôt le site antique sur le bord sud, se superpose à l'aménagement en bois au passage du chenal A et, plus à l'ouest, à la grande barre sableuse de crue qui occupe le chenal B. Celle-ci représente donc un événement chronologiquement bien calé entre la mise en place de la poutre, datée des années 20 apr. J.-C., et l'écroulement intégral du mur G qui marque la destruction du site vers les années 60 apr. J.-C.

\section{HISTOIRE GÉOARCHÉOLOGIQUE: MÉTAMORPHOSE ALLUVIALE}

Outre le fait qu'elle a permis de mettre au jour des structures originales (aménagement de chenaux, bassin piscicole implanté en milieu alluvial), la fouille géoarchéologique du site laténien et antique de Molesme, a fourni les éléments indispensable à une datation et à une quantification de l'impact anthropique sur le système socio-environnemental de la vallée de la Laigne. Le passage d'un état de plaine alluviale marécageuse à une plaine alluviale détritique constitue une véritable métamorphose ${ }^{11}$ du paysage que l'on peut désormais dater avec précision du début de l'époque gallo-romaine, soit entre les années 20 et 60 de notre ère. Cette transformation, à la fois rapide et irréversible de la vallée, fait écho à la première mise en place d'une gestion à grande échelle de l'ensemble du modeste bassin versant de la Laigne, suite à la construction de l'agglomération de Vertillum au ${ }^{\mathrm{er}}$ s. Celle-ci entraîna une déforestation et une mise en culture rapide du territoire agricole qui lui était lié. Faute de leur protection forestière, les sols

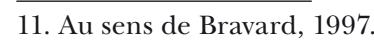




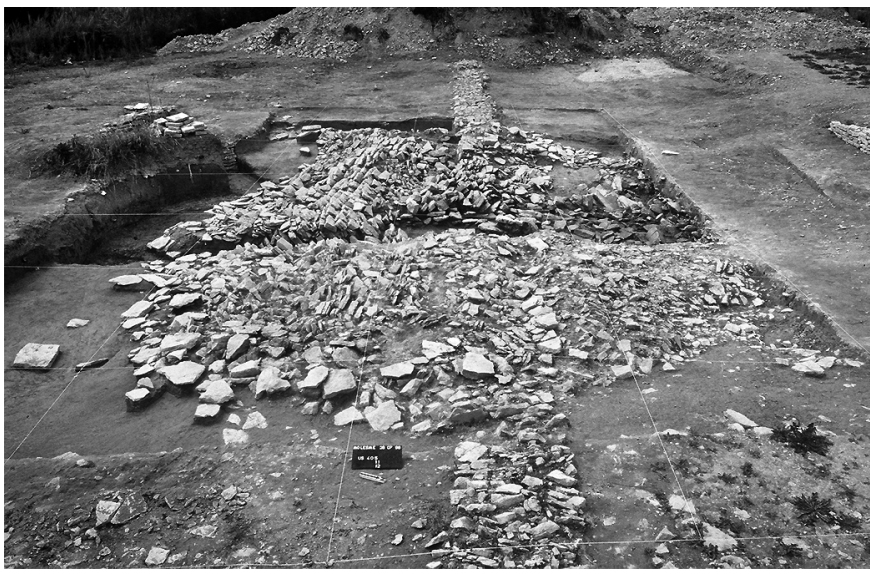

a

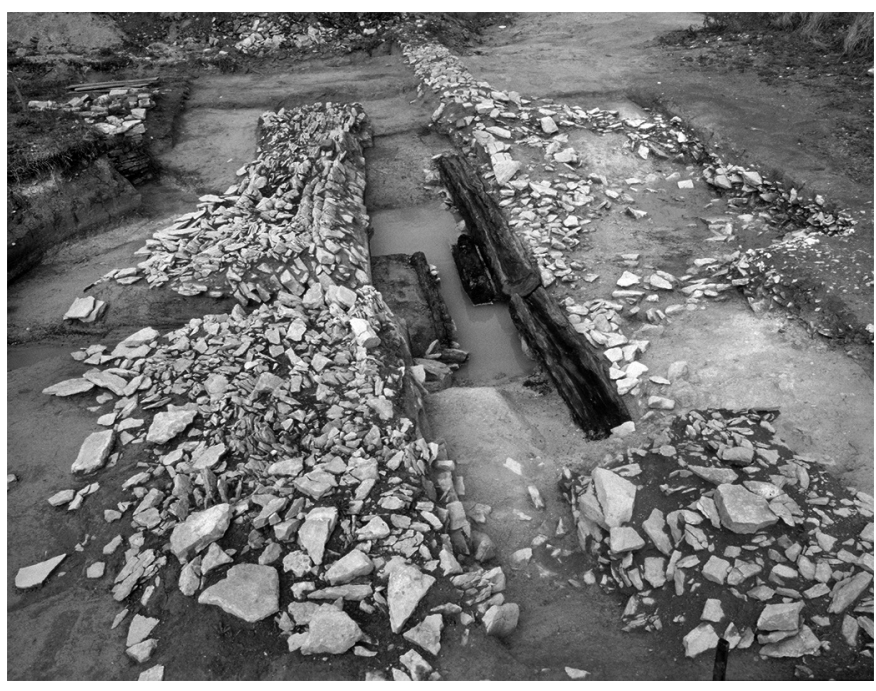

b

Fig. 11 - Photographies de l'avancement de la fouille du chenal A. En arrière-plan, la barre de crue sur laquelle est implanté le mur de direction est-ouest reliant le site antique à la rivière. Ce mur s'est effondré en un seul tenant dans le chenal A au milieu du $I^{\text {er }}$ s. La fouille de ce mur effondré a permis de mettre au jour une grande poutre barrant le chenal et mise en place aux environs des années 20 apr. J.-C. (clichés C. Petit, Université de Bourgogne).

des versants furent érodés en masse et vinrent recouvrir la plaine d'inondation à chaque nouvelle crue. Les «limons gris » qui supportent l'actuelle plaine d'inondation sont les produits de cette érosion. Cette importante sédimentation limoneuse a eu pour conséquence d'ensevelir la terrasse subboréale de bord de vallée et de niveler la plaine d'inondation. Dès lors, la surface sur laquelle les crues peuvent s'étendre augmente de façon très importante sans pour autant que le débit soit devenu plus important. Postérieurement au I ${ }^{\mathrm{er}}$ s., la sédimentation alluviale semble très faible, bien que le bassin versant ait subi d'autres impacts anthropiques (développement de la ville antique, changement de pratiques agricoles au haut Moyen Âge, gestion monastique médiévale et moderne) ou climatique (Petit Âge Glaciaire du XVII ${ }^{\mathrm{e}} \mathrm{s}$.). La raison en est probablement l'épuisement des stocks de matériaux disponibles issus des sols peu épais des plateaux et des versants calcaires.

\section{LA SÉDIMENTATION ALLUVIALE CONTRÔLÉE PAR DES FACTEURS ANTHROPIQUES ET HYDROCLIMATIQUES}

Dans la vallée de la Laigne, les changements de la dynamique sédimentaire intervenus depuis les trois derniers millénaires se sont enregistrés suivant les quatre modalités suivantes:

- une surface d'érosion majeure attribuable à l'époque subboréale. Dans tous les cas, elle est antérieure aux premiers indices palynologiques d'anthropisation, ainsi qu'aux premiers indices archéologiques repérés en vallée de Laigne. De toute évidence, cette érosion correspond à la dégradation hydroclimatique bien connue du SubboréalSubatlantique (entre $1500 \mathrm{BC}$ et $500 \mathrm{BC}$, voir Stuiver, Braziunas, 1993; Van Geel, Magny, 2002), dégradation également reconnue dans les phases transgressives des lacs jurassiens de Pluvis et du Bourget par M. Magny (1995) et l'avancée des glaciers (Haas et al., 1998);

- une forte augmentation du taux de sédimentation durant La Tène finale et le Haut-Empire. Il atteint alors respectivement 5 et $10 \mathrm{~mm} / \mathrm{an}$, tandis qu'au Subboréal, il n'excède pas $0,1 \mathrm{~mm} / \mathrm{an}$ ou s'avère même négatif (surface d'érosion subboréale) ;

- un changement dans la nature de la sédimentation dès le Haut-Empire. De carbonatée, la sédimentation autigène devient essentiellement détritique, suite à l'érosion agricole des versants;

- un enregistrement sédimentaire des événements des crues laténiennes et gallo-romaines. La plus marquée, datée de $40 \pm 20$ apr. J.-C., se place au début de la phase de sédimentation détritique. Il semblerait ainsi que, pendant la phase de forte sédimentation, la signature sédimentaire des crues soit amplifiée. De plus, les défrichements peuvent accentuer l'amplitude des crues (Cosandey, Robinson, 2000, p. 309 et 322).

Un changement de sédimentation intervenu à la fin du $\mathrm{I}^{\mathrm{er}}$ s. av. J.-C. a bien été perçu sur d'autres sites, en 
particulier dans la vallée du Rhône, mais il a été le plus souvent rapporté à des causes strictement hydroclimatiques : une dégradation climatique aurait en effet entraîné une augmentation importante du taux de sédimentation, ainsi que de la fréquence et de l'amplitude des crues (VérotBourrély et al., 1995; Berger, 1995; Provensal et al., 1999; Arlaud dir., 2000; Bruneton et al., 2001; Salvador et al., 2002; Trément et al., 2002 Berger, 2003). Or, il nous semble que les données paléoclimatiques obtenues pour cette époque ne traduisent pas de dégradation notable, bien au contraire (Stuiver, Braziunas, 1993; Reale, Dirmeyer, 2000; Reale, Shukla, 2000). En revanche, l'impact, dès l'époque augustéenne, de la romanisation sur l'organisation du paysage agraire de la Gaule est un fait largement démontré (Ferdière, 1988; Chouquer, Favory, 1991; Blin et al., 1999). L'extension, en particulier, des surfaces cultivées est à l'origine de la production sédimentaire (érosion des sols) qui crée les conditions de l'enregistrement des crues: seul un fort taux sédimentaire permet en effet de conserver la mémoire des crues anciennes. La survalorisation des crues gallo-romaines tient certainement plus à l'existence, pour cette époque, de matériaux sédimentaires d'enregistrement qu'à une réelle dégradation climatique. À l'inverse, l'absence de traces de crues pour les périodes antérieures ou postérieures ne démontre en rien leur absence; l'enregistrement fluvial des conditions climatiques dépend donc en premier lieu du degré d'anthropisation du milieu. Les aménagements des installations implantées en bordure de rivière piègent en effet de façon exceptionnelle des traces du fonctionnement des systèmes alluviaux.

Cet exemple n'est certainement pas isolé. L'interprétation climatique, souvent mise en avant, pourrait être nuancée par le facteur anthropique. À l'exception de quelques rares périodes, et dans des régions particulières, depuis deux millénaires, peut-être trois (et pourquoi pas plus dans certains endroits), l'homme est devenu le moteur principal - et souvent unique - des changements environnementaux. 


\section{BIBLIOGRAPHIE}

ADOLPHE J.-P.

1981 : Observations et expérimentations géomicrobiologiques et physicochimiques des concrétionnements carbonatés continentaux actuels et fossiles, thèse de Doctorat d'État, Université Paris-VI, $n^{\circ} 81-34$.

1989 : «Exemples de biosédimentologie: les tufs et les travertins », Bulletin du Centre de géomorphologie, 38, p. 29-33.

ARLAUD C. (DIR.) avec la collab. de LUROL J.-M., SAVAY-GUERRAZ S., VÉROT-BOURRÉLY A.

2000: Lyon, les dessous de la presqu'île, Lyon, Ministère de la Culture et de la Communication, Direction régionale des affaires culturelles (coll. Document d'archéologie en Rhône-Alpes, 20), $280 \mathrm{p}$.

Barral P., Guillaumet J.-P., Nouvel P. 2002: «Les territoires de la fin de l'âge du Fer entre Loire et Saône: les Éduens et leurs voisins. Problématique et éléments de réponse ", in GARCIA D., VERDIN F. (DIR.), Territoires celtiques, Actes du colloque de l'Association française pour l'étude de l'âge du Fer, 2000, Paris, éd. Errance, p. 272-296.

BERGER J.-F.

1995: "Facteurs anthropiques et naturels de l'évolution des paysages romains et protomédiévaux du Bassin valdainais (Drôme) ", in VAN DER LEEUW S. E. (DIR.), L'Homme et la dégradation de l'environnement, Actes des XV rencontres internationales d'archéologie et d'histoire d'Antibes, 20-22 oct. 1994, Sophia Antipolis, éd. ADCPA, p. 79-114.

2003: "Les étapes de la morphogenèse holocène dans le sud de la France ", in VAN DER LEEUW S. E., FAVORY F., FICHES J.-L. (DIR.), Archéologie et systèmes socio-environnementaux. Études multiscalaires sur la vallée du Rhône dans le programme "Archaeomedes", Paris, CNRS Éditions (coll. Monographie du CRA, 27), p. 87-167.

Berti L., Petit C., Wahlen P.

1998: Molesme en vallée de Laigne. Histoire et environnement d'un terroir entre Bourgogne et Champagne, Molesme, éd. de la Mairie de Molesme, $160 \mathrm{p}$.

\section{Bichet V., Campy M., Petit C.}

1997 : « Mise en évidence de l'impact anthropique sur la sédimentation associée aux sites archéologiques: apports, limites et perspectives ", in CHOUQUER G. (DIR.), Les Formes du paysage, t. 3, Paris, éd. Errance, p. 25-35.

\section{BICHET V., CAMPY M.,}

BUONCRISTIANI J.-F., Di GIOVANNI C., MEYBECK M., RICHARD H.

2002 : «Évolution quantitative de la production sédimentaire d'un bassin versant carbonaté au cours du Tardiglaciaire et de l'Holocène: l'exemple de la haute vallée du Doubs (France) ", in BRAVARD J.-P., MAGNY M. (DIR.), Histoire des rivières et des lacs de Lascaux à nos jours, Paris, éd. Errance, p. 161-170.

Blin O., Gebhardt A., Allenet G., BERNARD V., BOYER F., DIÉTRICH A., LEROYER C., MARGUERIE D., MATTERNE V., SEYRIESSOL K., ZWIERZINSKI E.

1999 : «Impact anthropique et gestion du milieu durant l'Antiquité: l'approche paloenvironnementale pluridisciplinaire du site "La ferme d'Ithe" à JouarsPonchartrin (Yvelines)", Les Nouvelles de l'archéologie, 78, p. 45-56.

\section{BRAVARD J.-P.}

1997 : «Géoarchéologie des vallées alluviales de Rhône-Alpes depuis le Tardiglaciaire ", in BRAVARd J.-P., PRestreau M. (DIR.), Dynamique du paysage: entretiens de géoarchéologie, Actes de la table ronde, Lyon, 17-18 nov. 1995, Lyon, Ministère de la Culture et de la Communication, Direction régionale des affaires culturelles (coll. Documents d'archéologie en Rhône-Alpes, 15), p. 129-150.

BROWN A. G.

1997: Alluvial Geoarchaeology: Floodplain Archaeology and Environnemental Change, Cambride, Cambridge University Press, $377 \mathrm{p}$.

\section{BRUNETON H., ARNAUd-Fassetta G.,} Provansal M., Sistach D.

2001 : «Geomorphological evidence for fluvial change during the Roman period in the lower Rhône valley (Southern France) ", Catena, 45 , p. 287-312.

\section{CAMPY M., MACAIRE J.-J.}

2003 (2éd.) : Géologie de la surface. Érosion, transfert et stockage dans les environnements continentaux, Paris, Dunot, 440 p.
ChafeTZ H. S., FOLK R. L.

1984 : « Travertines: depositional morphology and the bacterially constructed constituents ", Journal of Sedimentary Petrology, 54, p. 289-316.

\section{Chaume B.}

2001 : Vix et son territoire à l'âge du Fer. Fouilles du Mont-Lassois et environnement du site princier, Montagnac, Monique Mergoil (coll. Protohistoire européenne, 6), $643 \mathrm{p}$.

ChOUQuer G., FAVORY F.

1991: Les Paysages de l'Antiquité, Paris, éd. Errance, $243 \mathrm{p}$.

\section{Chrétien J.}

2000: Référentiel pédologique de Bourgogne à 1/250 000. Régions naturelles, pédopaysages et sols de la Côte-d'Or, Paris, INRA, 194 p.

\section{Columelle}

2002: De l'Agriculture, trad. Saboureux de la Bonnetterie et M. Nisard, Paris, éd. Errance, 336 p.

\section{COSANDEY C., ROBINSON M.}

2000 : Hydrologie continentale, Paris, Armand Colin, $360 \mathrm{p}$

Courty M.-A., Goldberg P., MACPHAIL R. I.

1989 : Soils and Micromorphology in Archaeology, Cambridge, Cambridge University Press, $344 \mathrm{p}$.

FERDIÈRE A.

1988: Les Campagnes de la Gaule romaine -ILes Hommes et l'environnement en Gaule rurale (52 av. J.-C.-486 apr. J.-C.), Paris, éd. Errance, $301 \mathrm{p}$.

\section{FREYTET P.}

1989 : "Contribution à l'étude des tufs du Bassin de Paris; typologie des édifices tuffacés (stromatolitiques) des chenaux fluviatiles (aspect mascroscopique)", Bulletin du Centre de géomorphologie, 38, p. 9-28.

\section{Freytet P., Plet A.}

1996 : « Modern freshwater microbial carbonates: the Phormidium stromatolites (tufatravertine) of southeastern Burgundy (Paris Basin, France) ", Facies, 34, p. 219-238.

\section{GAUThier E.}

2001 : Évolution de l'impact de l'homme sur la végétation du massif jurassien au cours des quatre 
derniers millénaires. Nouvelles données palynologiques, thèse de Doctorat, Besançon, Université de Franche-Comté, 250 p.

\section{Gauthier E., Richard H., Petit C.}

2006: Analyses polliniques du marais de l'Abîme à Molesme (Côte-d'Or, France), in PETIT C. (DIR.), Occupation, gestion et paléoenvironnement des plaines alluviales de l'âge du Fer à l'Antiquité, Actes de la table ronde de Molesme, 17-18 sept. 1999, Besançon, Presses universitaires franc-comtoises (coll. Annales littéraires de l'université de Besançon, 786, série Environnement, sociétés et archéologie, 8), p. 41-45.

\section{GeURTS A. M.}

1976 : " Genèse et stratigraphie des travertins de fond de vallée en Belgique ", Acta geographica lovaniensia, 16, p. 1-66.

\section{GOGUEY R.}

2003: "Archéologie aérienne en pays chatillonnais ", Dossier de l'archéologie, 284, p. $52-61$.

HAAS J. N., RICHOZ I., TINNER W., WICK L. 1998 : « Synchronous Holocene climatic oscillations recorded on the Swiss plateau and the timberline in the Alps ", The Holocene, 98, p. 301-304

INGARGIOLA J.-F., JACQUIN T., AMIOT M., PASCAL A., RAT P., THIERRY J.,

MARION M., TINETTE R.

1989 : Les Ressources en eaux du Châtillonnais, Étude publiée par le Conseil général de la Côte-d'Or, 67 p.

\section{JOFFROY R.}

1960 : L'Oppidum de Vix et la civilisation hallstatienne dans l'est de la France, Paris, Les Belles Lettres (coll. Publications de l'université de Dijon, 20), 210 p.

JOUIN M., MÉNIEL P.

2001 : "Les dépôts d'animaux et le fanum gallo-romains de Vertault (Côte-d'Or) », Revue archéologique de l'Est, 50, p. 119-216.

\section{JOURNAUX A.}

1963: Le Rôle du vent dans la formation des dépôts cryoclastiques de pente, Paris, Comptes rendus de l'Académie des sciences, 256, p. 3724-3725.

\section{LÉCOLLE F., LÉTOLLE R.}

1989 : «Paléotempératures déduites de la composition isotopique des tests de gastéropodes terrestres et des travertins de la vallée de la Seine ", Bulletin du Centre de géomorphologie, 38, p. 79-92.
LenOIR G., PAGNey P.

1982 : «Climatologie du Châtillonnais », Cahiers du Centre d'études régionales de Bourgogne. Université de Dijon, 1, p. 29-35.

LOREAU J.P., THIERRY J.

1975 : Carte géologique au 1/50 000, Les Riceys, carte $\mathrm{n}^{\circ} 370$, Service géologique national, BRGM éditions.

\section{MAGNY M.}

1995: Une Histoire du climat: des derniers mammouths au siècle de l'automobile, Paris, éd. Errance, 176 p.

MANGIN J.-M., MANGIN-JOUIN M.

1994 : «Vertault-Vertillum », in BÉNARD J., MANGIN M., GOGUeY R., ROUSSEl L. (DIR.), Les Agglomérations antiques de Côte-d'Or, Paris, Les Belles Lettres (coll. Annales littéraires de l'université de Besançon, 522, série Archéologie, 39), p. $91-105$.

\section{MARANSKI D.}

1997: «Le Châtillonnais au premier âge du Fer: ensembles funéraires, coupures chronologiques et organisation du territoire ", Revue archéologique de l'Est, 48, p. 51-88.

\section{MARION M.}

1982 : «Hydrologie du Châtillonnais. Principales caractéristiques du régime des cours d'eau et essai de bilan ", Cahiers $d u$ Centre d'études régionales de Bourgogne. Université de Dijon, 1, p. 45-62.

MARTIN S., MAGNIN F.

2002: «Caractérisation des perturbations anthropiques dans la séquence holocène de l'Ubac (Goult, Vaucluse), à partir de la malacofaune ", in RICHARD H., VIGNOT A. (DIR.), Équilibres et ruptures dans les écosystèmes durant les 20 derniers millénaires en Europe de l'Ouest, Actes $d u$ colloque international de Besançon, sept. 2000, Besançon, Presses universitaires franc-comtoises (coll. Annales littéraires de l'université de Besançon, 730, Série environnement, sociétés et archéologie, 3), p. 205-212.

\section{Messerli B., Grosjean M., Hofer T.,} NUNEZ L., PFISTER G.

2000 : «From nature-dominated to humandominated environmental changes", Quaternary Sciences Reviews, 19 p. 459-479.

Orth P., Pastre J.-F., Gauthier A. LIMONDIN-LOZOUET N., KUNESH S.

2004: «Les enregistrements morpho- sédimentaires et biostratigraphiques des fonds de vallée du bassin-versant de la Beuvronne (Bassin parisien, Seine-etMarne, France) : perceptions des changements climato-anthropiques à l'Holocène ", Quaternaire, 15, 3, p. 285-298.

PASTRE J.-F., LEROYER C., LIMONDINLOzouey N., ORTh P., Chaussé C., FonTUGNe M., GaUTHiER A., KUneSch S., LE JeUne Y., SAAD M.-C. 2002: «Variations paléoenvironnementales et paléohydrologiques durant les 15 derniers millénaires: les réponses morphosédimentaires des vallées du Bassin parisien (France) ", in BRAVARD J.-P., MAGNY M. (DIR.), Histoire des rivières et des lacs de Lascaux à nos jours, Paris, éd. Errance, p. 29-44.

\section{PAUTRAT Y.}

2002: "La Préhistoire du Chatillonnais", Dossier d'archéologie, 284, p. 12-18.

PEDley H. M.

1990: "Classification and environmental models of cool freshwater tufas", Sedimentary Geology, 68, p. 255-274.

PeTit C., Wahlen P

2002 :« Molesme "Sur-les-Creux", desbanquets gaulois aux poissons gallo-romains", Dossier d'archéologie, 284, p. 90-97.

2004: «Une image de la religion des Celtes: la statue gauloise découverte à Molesme », Dossier d'archéologie, Bourgogne du Paléolithique au Moyen Âge, hors série $\mathrm{n}^{\mathrm{o}}$ II, déc. 2004, p. 29

Petit C., WAHLEN P., ARRignON J.

2005: «Le bassin gallo-romain de Molesme (21) : questions et perspectives de recherche sur les viviers antiques d'eau douce ", in PETIT C. (DIR.), Occupation et gestion des plaines alluviales dans le nord de la France de l'âge du Fer à l'époque galloromaine, Actes de la table ronde de Molesme, 17-18 sept. 1999, Besançon, Presses universitaires franc-comtoises (coll. Annales littéraires de l'université de Besançon, 786, série Environnement, sociétés et archéologie, 8), p. 47-62.

Petit C. ET AL.

2005: «Approche géoarchéologique de la vallée de la Laigne ", in PETIT C. (DIR.), in PETIT C. (DIR.), Occupation et gestion des plaines alluviales dans le nord de la France de l'âge du Fer à l'époque gallo-romaine, Actes de la table ronde de Molesme, 17-18 sept. 1999, Besancon, Presses universitaires franccomtoises (coll. Annales littéraires de 
l'université de Besançon, 786, série Environnement, sociétés et archéologie, $8)$, p. $23-40$

PROVANSAL M., BERGER J.-F., BRAVARD J.-P., SAlVAdOR P.-G., ARNAUd-FasseTa G., BRUNETON H., VÉROT-BOURRÉLY A.

1999 : «Le régime du Rhône dans l'Antiquité et au haut Moyen Âge ", in LEveAU P. (DIR.), «Le Rhône romain: dynamiques fluviales, dynamiques territoriales ", Gallia, 56, p. 13-32.

\section{PUISSÉGUR J.-J.}

1976: Mollusques continentaux quaternaires de Bourgogne, Mémoire de géologie de l'université de Bourgogne, $241 \mathrm{p}$.

\section{REALE O., DIRMEYER P.}

2000: "Modeling the effects of vegetation on mediterranean climate during the Roman Classic period. Part I: Climate history and model sensivity ", Global and Planetary Change, 25, p. 163-184.

\section{REALE O., SHUKLA J.}

2000: "Modeling the effects of vegetation on mediterranean climate during the Roman Classic period. Part II: Model simulation ", Global and Planetary Change, 25 , p. $185-214$

\section{ROLley C.}

2003: «La tombe de Vix dans l'histoire de la recherche ", in ROLLEY C. (DIR.), $L a$ Tombe princière de Vix, Paris, Picard, 2 vol., p. 11-20.

\section{SALVADOR P.-G., VÉROT-BOURRÉly A.,} BRAVARD J.-P., FrANC O., MACÉ S.

2002: «Les crues du Rhône à l'époque romaine dans la région lyonnaise ", in BRAVARD J.-P., MAGNY M. (DIR.), Histoire des rivières et des lacs de Lascaux à nos jours, Paris, éd. Errance, p. 215-221.

\section{STUIVER M., BRAZIUNAS T. F.}

1993: «Sun, ocean, climate and atmospheric ${ }^{14} \mathrm{CO}_{2}$ : an evaluation of causal and spectral relationships ", The Holocene, p. 289-305.

STUiver M., Reimert P. J., BARd E., BECK J. W., BURR G. S., HUGHEN K. A., KROMER B., McCormaC G., VAN DER Plicht J., SPURK M.

1998: "Radiocarbon age calibration, 24,000-0 cal. BP », Radiocarbon, 40, 3, p. 1041-1083.

Trément F., Ballut C., MennessierJouAnNet C., ARgant J.

2002: "Mutations environnementales et systèmes socio-économiques en Grande Limagne (Massif central français) de l'âge du Fer au Moyen Âge ", in RICHARD H., VIGNOT A. (DIR.), Équilibres etruptures dans les écosystèmes durant les 20 derniers millénaires en Europe de l'Ouest, Actes du colloque international de Besançon, sept. 2000, Besançon, Presses universitaires franccomtoises (coll. Annales littéraires de l'université de Besançon, 730, série environnement, sociétés et archéologie, 3), p. $269-279$.

VAN Der Leeuw S. E., Favory F.

FICHES J.-L. (DIR.)

2003 : Archéologie et systèmes socio-environnementaux. Études multiscalaires sur la vallée du Rhône dans le programme "Archaeomedes", Paris, CNRS Éditions (coll. Monographie du CRA, 27), $403 \mathrm{p}$.

\section{VAN GEEL B., MAGNY M.}

2002: «Mise en évidence d'un forçage solaire du climat à partir de données paléoécologiques et archéologiques: la transition Subboréal-Subatlantique ", in RICHARD H., VIGNOT A. (DIR.), Équilibres et ruptures dans les écosystèmes durant les 20 derniers millénaires en Europe de l'Ouest, Actes du colloque international de Besançon, sept. 2000, Besançon, Presses universitaires franc-comtoises (coll. Annales littéraires de l'université de Besançon, 730, série Environnement, sociétés et archéologie, 3), p. 107-122.

VAUDOUR J.

1986 : « Travertins holocènes et pression anthropique ", Méditerranée, 57, 1-2, p. 25-30.

\section{VÉrot-Bourrély A., ARGANT J.} BRAVARD J.-P., CHAIX L.

1995: «Le paléoenvironnement du site de Gorge de Loup (Lyon 9e) : signes matériels de la dégradation d'après l'étude interdisciplinaire du gisement ", in VAN DER LEEUW S. E. (DIR.), L'Homme et la dégradation de l'environnement, Actes des $X V^{P}$ rencontres internationales d'archéologie et d'histoire d'Antibes, 20-22 oct. 1994, Sophia Antipolis, éd. ADCPA, p. 43-77.

\section{WEISROCK A.}

1986 : «Variations climatiques et périodiques de la sédimentation carbonatée à l'Holocène. L'âge des dépôts ", Méditerranée, 57, 1-2, p. 165-167.

\section{ZEEPVAT R. J.}

1988: "Fishponds in Roman Britain ", in Aston M. (ED.), Medieval Fish, Fisheries and Fishponds in England, Oxford (coll. British Archaeological Report, british series, 182 [i] et [ii]), p. 17-27. 